\title{
Intraspecific variation in immune gene expression and heritable symbiont density
}

Holly L. Nichols, ${ }^{1}$ Elliott B. Goldstein, ${ }^{1}$ Omid Saleh Ziabari, ${ }^{2}$ Benjamin J. Parker ${ }^{1,}$

${ }^{1}$ Department of Microbiology, University of Tennessee, Knoxville, TN, 37996, USA

${ }^{2}$ Department of Biology, University of Rochester, Rochester, NY 14627, USA

ORCIDs: HLN: 0000-0003-2158-1767, EBG: 0000-0001-7651-0486, OSZ: 0000-0003-1949-9035, BJP: 0000-0002-0679-4732

*author for correspondence: 1311 Cumberland Avenue, Knoxville, TN, USA 37996, bjp@utk.edu, +1 865 9749818

Classification: Biology > Evolution

Keywords: Symbiosis, Genetic variation, Gene expression, Host-microbe interactions, Immunity, Pea aphid 
ABSTRACT

Host genetic variation plays an important role in the structure and function of heritable microbial communities. Recent studies have demonstrated that insects use immune mechanisms to regulate heritable symbionts. Here we test the hypothesis that variation in symbiont density within hosts is linked to intraspecific differences in the immune response to harboring symbionts. We show that pea aphids (Acyrthosiphon pisum) harboring the bacterial endosymbiont Regiella insecticola (but not all other species of symbionts) suppress expression of key immune genes. We then functionally link immune suppression with symbiont density using RNAi. The pea aphid species complex is comprised of multiple reproductively-isolated host plant-adapted populations. These 'biotypes' have distinct patterns of heritable symbiont infections: for example, aphids from the Trifolium biotype are strongly associated with Regiella. Using RNAseq, we compare patterns of gene expression in response to Regiella in aphid genotypes from multiple biotypes, and we show that Trifolium aphids experience no immune gene suppression from Regiella and host symbionts at lower densities. We then generated F1 hybrids between two biotypes and found that symbiont density and immune suppression are both intermediate in hybrids. We suggest that in this system, Regiella symbionts are suppressing aphid immune mechanisms to increase their density, but that some hosts have adapted to prevent immune suppression in order to control symbiont numbers. The specific immune mechanisms suppressed by Regiella have been previously demonstrated to combat pathogens in aphids, and thus this work highlights the immune system's complex dual role in interacting with both beneficial and harmful microbes.

\section{AUTHOR SUMMARY}

42

Heritable microbes are found in most insects including agriculturally and medically relevant pests.

Explaining the variation in the distribution and abundance of symbionts in natural populations is critical to understanding these interactions. This work contributes to our mechanistic understanding of an important model of host-microbe symbiosis and suggests more broadly that variation in insect immune responses plays a role in intraspecific variation in host-symbiont interactions. Our work also suggests that vertically and provide a clear benefit to their hosts. 
INTRODUCTION:

Most insects harbor heritable microbes that have important effects on host fitness (1-3). A key aspect of these symbioses is variation. Across species, host taxonomy has been shown to play a role in structuring heritable microbial communities (4-6). Within species, microbes referred to as 'facultative' symbionts are not found in all individuals, and symbiont frequencies are subject to selection on the relative costs and benefits of harboring microbes $(7,8)$. In addition to microbiome composition, hosts vary in other aspects of symbioses like the density of microbial infections (9). For example, two closely-related species of Nasonia wasps vary in the density at which they harbor Wolbachia bacteria, and this variation is due to a single gene that somehow suppresses maternal transmission of bacteria (10). Except for a few examples, little is known about the mechanisms that underlie variation in heritable symbioses or the evolutionary genetics of these interactions (11).

Invertebrate immune systems have been shown to play a direct role in mediating interactions with heritable microbes. In grain weevils, for example, an antimicrobial peptide acts to confine mutualistic symbionts to specialized cells called bacteriocytes (12), and silencing expression of immune pathways allows symbionts to escape bacteriocytes (13). Other studies have found more complex interactions between pathogens, the immune system, and vertically-transmitted symbionts: In Drosophila melanogaster, for example, activation of the Toll and IMD immune pathways results in an increase in density of Spiroplasma symbionts (14) (and see similar examples in mosquitos (15) and tsetse flies (16)), suggesting in some systems the immune system can promote beneficial microbes by inhibiting other microbes.

Immune genes are among the fastest evolving genes in eukaryotic genomes (17-19), and natural populations harbor genetic variation in immune mechanisms (20). Given the importance of the immune system in regulating insect symbioses, it seems likely that variation in immune mechanisms contributes to variation in symbiont density among hosts. This hypothesis is complicated, however, because our models for the maintenance of genetic variation in immune systems are based on antagonistic coevolution between hosts and pathogenic microbes $(21,22)$. Heritable symbiont infections are thought to spread through host populations because the fitness interests of host and microbe are generally aligned, and many symbionts have been shown to benefit their hosts for example by providing protection from pathogens (reviewed in (23)). But symbionts can impose costs on their hosts (e.g. (24)), and selection may favor the loss of symbionts in certain contexts (25). In addition, within-host selection might lead to a separation of the fitness interests of hosts and microbes. For example, a mutation in a symbiont genome that increases symbiont density might increase the likelihood of symbiont transmission but come at the expense of host fitness. Hosts, in turn, could evolve greater control over symbiont numbers in an 
ongoing arms-race for control over a symbiosis. It is unclear, however, whether the 'arms-race' dynamics underlying host-pathogen coevolution also govern the evolutionary interactions between immune systems and beneficial microbes.

The pea aphid (Acyrthosiphon pisum) is an important insect-symbiont model system (26). The pea aphid species complex is composed of multiple reproductively-isolated populations adapted to live on different host plants within the family Fabaceae. These 'biotypes' are genetically differentiated and are estimated to have radiated onto different host plants $\sim 500,000$ years ago (27) (but see (28)). In addition to obligate intracellular bacteria called Buchnera aphidicola, aphids can harbor several species of facultative symbionts. Multiple studies have found that facultative symbionts are non-randomly distributed across aphid biotypes (29-32). For example, Regiella insecticola (which confers protection against fungal pathogens to its host (33-35)) is strongly associated with aphids from the Trifolium spp. (clover) biotype across continents. A number of studies have explored whether the strong association between Trifolium biotype aphids and Regiella is due to improved host plant use with mixed results (36-39). Alternatively, this association could be due to the risk of exposure to fungal pathogens (though see (40)), to historical contingency (though see (41)), or to host and/or symbiont genetic mechanisms. This system therefore provides a useful natural laboratory to study host-microbe adaptation across multiple environments within a single species.

In this study, we show that immune mechanisms play a role in intraspecific variation in the density of a heritable bacterial symbiont. We first demonstrate that pea aphids that harbor Regiella (but not all other species of symbionts) sharply downregulate key innate immune genes, and that experimental suppression of the immune gene phenoloxidase via RNAi increases symbiont density. We then measure immune suppression across aphids from multiple biotypes and find that aphids from Trifolium spp. do not experience immune suppression and harbor symbionts at relatively low density. By performing an F1 cross between genotypes from two biotypes we find that hybrid aphids show intermediate symbiont densities and immune suppression, shedding light on the role of host genetic variation and the genomic architecture of this variation. We discuss these findings in light of the biology of this system and suggest that antagonistic coevolution between 'beneficial' microbes and their hosts can shape host-symbiont associations.

RESULTS:

Hosting some symbiont species leads to suppressed host immune gene expression. Aphid lines reproduce parthenogenetically under summer conditions, and facultative bacteria can be introduced into 
or removed from host lines. We established lines that have the same aphid host genotype (LSR1, collected from Medicago sativa (42)) with two different strains of Regiella: one from each of the two main phylogenetic clades of Regiella found in natural populations of pea aphids (43). Regiella strain .LSR was originally collected with the LSR1 aphid genotype and is a representative of Regiella 'Clade 1,' and Regiella strain .313 is from 'Clade 2' (44). We maintained aphids in the lab for four generations after symbiont establishment and then measured Regiella densities using quantitative PCR (qPCR). We have found previously that 'Clade 2' Regiella establish at higher densities in hosts than do 'Clade 1' strains, independent of host genotype (45). Consistent with this previous work, Regiella strain .313 established in aphid genotype LSR1 at a significantly higher density $(3.5 X)$ than Regiella strain .LSR $(t=5.1, p=0.006$, Figure 1A).

A Symbiont strain density

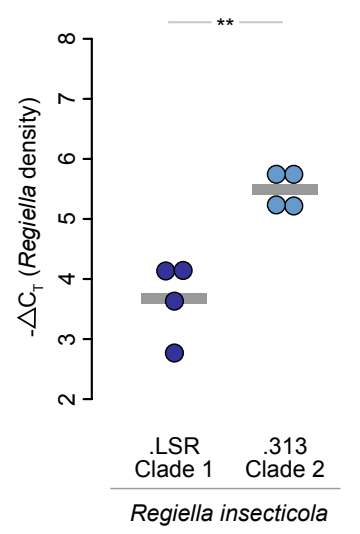

B $\quad$ LSR1 +.LSR (Regiella Clade 1)

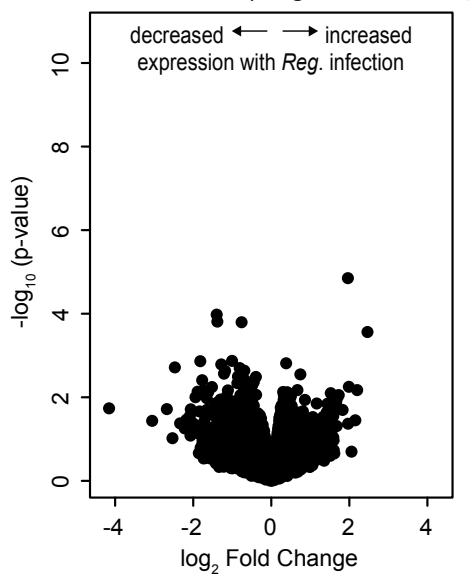

C LSR1 +.313 (Regiella Clade 2)

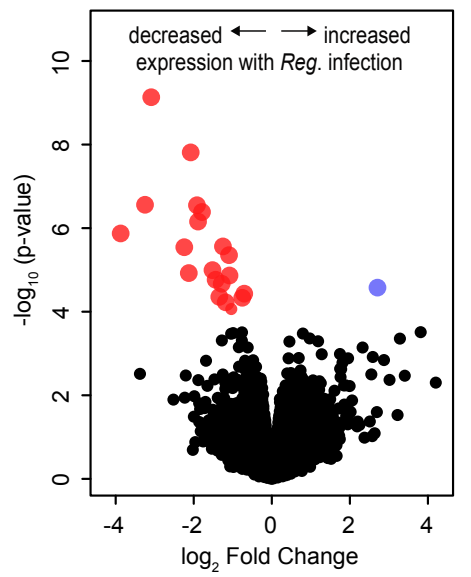

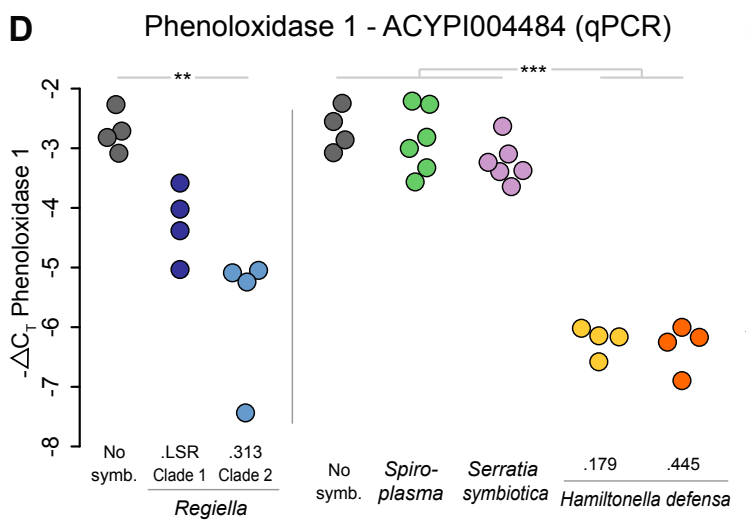

E

Hemocytin - ACYPI003478 (qPCR)
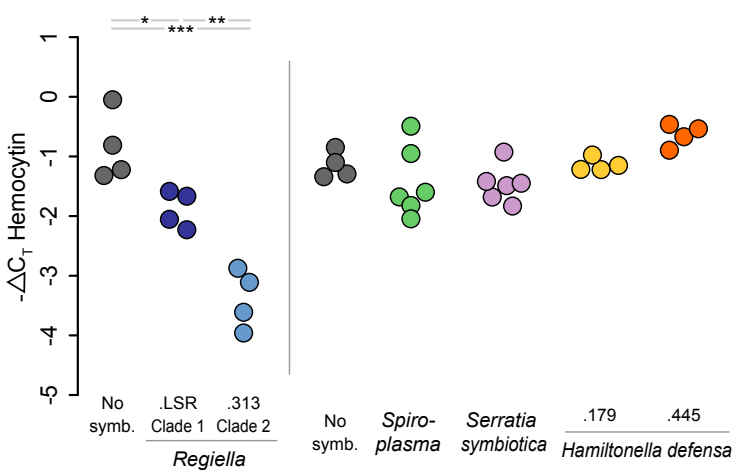

Figure 1: Effects of hosting secondary symbionts on aphid gene expression. A: Regiella density of lines harboring clade 1 and clade 2 Regiella. The $y$-axis shows the $-\Delta \mathrm{C}_{\mathrm{T}}$ values which can be interpreted on a $\log _{2}$ scale. B\&C: Volcano plots of RNAseq data comparing expression of each expressed gene in the aphid genome, represented by a point in each figure, between aphids with and without Regiella. A and $B$ show this analysis for aphids with a Regiella strain from clade 1 (strain .LSR) and clade 2 (strain .313), respectively. The $x$-axes show the $\log _{2}$ fold change for each gene, and the $y$-axis shows the significance of expression. Blue and red dots represent genes that were significantly up- or downregulated, respectively, at an FDR < 0.05. D: qPCR analysis of gene expression of Phenoloxidase 1 
without symbionts, and colored points show those with symbiont infections 4 generations after symbiont establishment. The different symbiont species and strains are shown along the bottom of the figure. The $y$-axis shows the $-\Delta C_{T}$ values of expression, which can be interpreted on a $\log _{2}$ scale. Statistical significance among species or strains is shown along the top of the figure; the two experiments, separated by a grey line, were analyzed separately. E: Same as panel D but for the gene hemocytin (ACYPI003478).

We then used RNAseq to measure how harboring Regiella influences aphid gene expression by comparing these lines with symbiont-free aphids that were sham-injected. We sequenced cDNA made from mRNA for 4 biological replicates of each line (where a biological replicate represents an independent aphid line injected with symbionts or sham-injected). Overall, harboring Regiella strain .LSR did not significantly alter expression of any genes in the aphid genome (FDR < 0.05; Figure 1B), while strain .313 led to significantly decreased expression of 19 genes and upregulation of 1 (FDR $<0.05$; Figure 1C).

The 20 genes with altered expression included key innate immune system genes (Table S1). In particular, the two copies of phenoloxidase in the pea aphid genome (referred to here as PO1 and PO2) were downregulated in the presence of Regiella. Also downregulated was a gene called hemocytin, which encodes a protein released by immune cells that plays a role in immune cell aggregation $(46,47)$. Other differentially expressed genes included a toll-like receptor and a putative lipopolysaccharide recognition protein (Table S1).

We used qPCR to directly compare expression of two immune genes between lines harboring the two Regiella strains and to confirm our RNAseq results. PO1 was significantly downregulated in lines harboring Regiella strain .313 (Figures 1D \& 1E, left panels). Hemocytin was significantly downregulated in lines harboring either symbiont, and the magnitude of this change was significantly stronger for aphids harboring strain .313 than those with strain .LSR (Figures 1D \& 1E, left panels).

Next, we established aphid lines that harbored several additional species of aphid facultative endosymbionts as above, and we looked for changes in PO1 and hemocytin expression. Spiroplasma and Serratia symbiotica did not alter expression of either gene, but two strains of Hamiltonella defensa significantly downregulated PO1 expression but not hemocytin (post-hoc tests, Table S2; Figures 1D \& $1 \mathrm{E}$, right panels). Like Regiella, the specific strain of Spiroplasma (.161) we used was found in previous work to protect against fungal pathogens (48), but Serratia and Hamiltonella have been found not to influence fungal resistance $(35,49,50)$. These results therefore suggest that the changes we identify in immune gene expression do not reflect the mechanism by which Regiella confers protection to aphids against fungal pathogens, which is currently unknown.

Immune gene expression influences symbiont density during development. We studied the function of immune gene expression on Regiella densities using RNA interference (RNAi) (51). We knocked-down expression of PO1 early in development and measured the effects on symbiont density. 
182

183

184

185

186

187

188

189

190

191

192

193

194

195

196

197

198

199

200

201

202

203

204

205

206

207

208

209

210

211

212

213

214

215

216

We synthesized dsRNA for PO1 and a lacZ control, and we injected $\sim 100$ ng of dsRNA in salt buffer into 1-day-old aphids. Aphids harbored either Regiella strain .LSR or .313. We then sampled aphids at two timepoints: at $72 \mathrm{hrs}$ after injection and after aphids had become adults ( 8 days after injection).

Injection with $P O 1$ dsRNA reduced $P O 1$ expression, on average, by $\sim 60 \%$ at $72 \mathrm{hrs}$ (2-way ANOVA; Treatment: $F=9.8, p=0.009$; Figure $2 A$ ). At this early timepoint, we found no significant difference between aphids harboring Regiella strain .LSR vs strain .313 in $P 01$ expression (Strain: $F=0.10, p=$ 0.75; Figure 2A). By the time aphids became adults (8 days after injection), PO1 expression in PO1 dsRNA injected aphids was still reduced by $\sim 60 \%$ compared with controls (Treatment: $F=3.6, p=0.02$; Figure 2A). By this later timepoint, aphids harboring the two symbiont strains had diverged in expression as found above (Strain: $F=11.5, p=0.004$; Figure $2 A$ ). Note that we dissected out and removed developing embryos only from the adult samples before nucleic acid extraction, so we do not directly compare expression in the $72 \mathrm{hrs}$ vs. adult samples, but qualitatively PO1 expression increased during development (Figure 2A).

PO1 knockdown led to a 59\% and 2\% increase in Regiella strain .LSR and .313 density at $72 \mathrm{hrs}$, respectively (2-way ANOVA; Treatment: $F=4.7, p=0.05$; Figure 2B). Regiella density in aphids harboring strain .LSR vs .313 also differed significantly (Strain: $F=245, p<0.0001$; Figure 2B), suggesting that strain-level differences in symbiont density are present even at this early developmental timepoint. The increase in Regiella density due to PO1 knock-down persisted to adulthood (Treatment: $F$ $=5.7, p=0.03$, Figure 2B), with PO1 dsRNA injection increasing Regiella density by $48 \%$ and $40 \%$ in aphids harboring Regiella strains .LSR and .313, respectively. As we found above, the density of strain .313 was higher than strain .LSR in adult aphids (Strain: $F=145, p<0.0001$; Figure 2B). Together, these results show that knockdown of PO1 increases Regiella density over development.

\section{Symbiont density is not impacted by immune gene knock-down later in development. We} performed a similar experiment studying the effect of RNAi on symbiont density, but injected dsRNA into adult aphids rather than $1^{\text {st }}$ instars. We injected $\sim 1 \mu \mathrm{g}$ of dsRNA synthesized from PO1 or hemocytin into adult (9 day old) aphid genotype LSR1 aphids infected with Regiella strain .LSR. We measured gene expression and Regiella density at $72 \mathrm{hrs}$ after injection. This led to a $\sim 69 \%$ and $\sim 82 \%$ reduction in expression of $P O 1$ and hemocytin, respectively (t-tests; $P O 1: t=-2.3, p=0.05$; hemocytin: $t=-7.3, p<$ 0.0001; Figures $1 \mathrm{C}$ and 1D). We note that injection with dsRNA from $P O 1$ had no effect on expression of the other copy of phenoloxidase in the aphid genome (PO2: $\mathrm{t}=-0.91, \mathrm{p}=0.38$; Figure $1 \mathrm{C}$ ), demonstrating that our phenoloxidase RNAi assay is specific to $P 01$ as designed. Knockdowns had no effect on Regiella density in aphids injected as adults (ANOVA; Treatment: $F=0.80, p=0.46$; Figure $1 \mathrm{E})$. 
A $\quad 1^{\text {st }}$ instar RNAi knockdown of $P O 1$ (ACYPI004484)

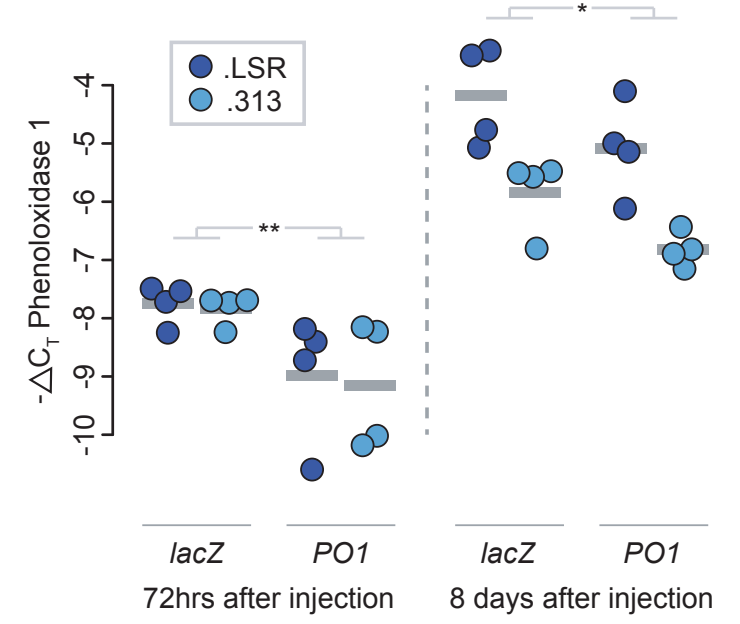

B Regiella density after knockdown

(1 ${ }^{\text {st }}$ instar dsRNA injections)

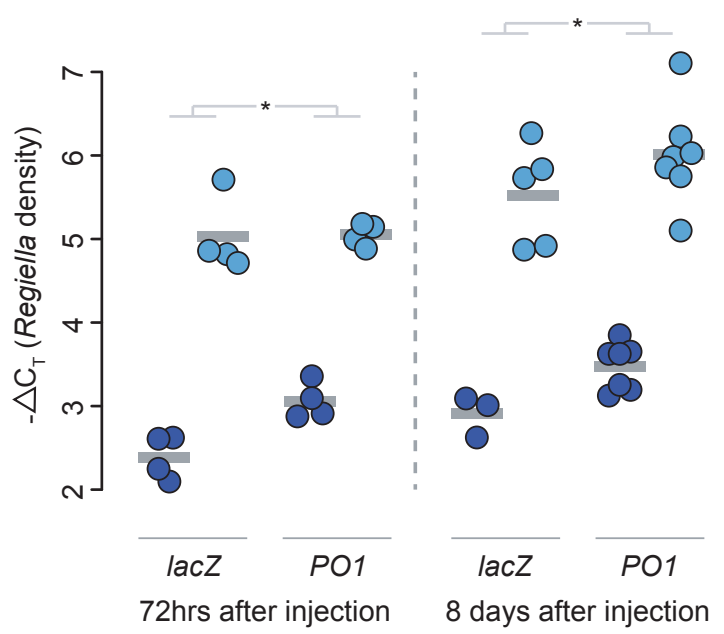

\section{Adult RNAi knock-down of PO1 (ACYPI004484)}
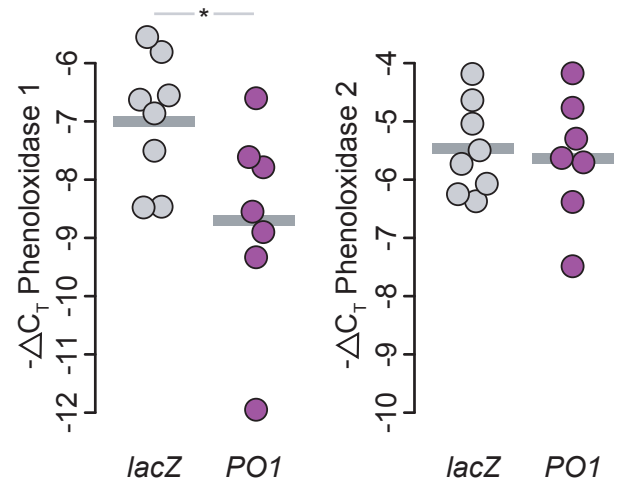
as above.
D Adult RNAi Knockdown of Hemocytin (ACYPI003478)

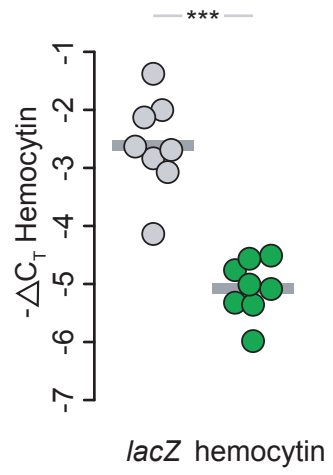

\section{E Regiella density after knockdown (adult dsRNA injections)}

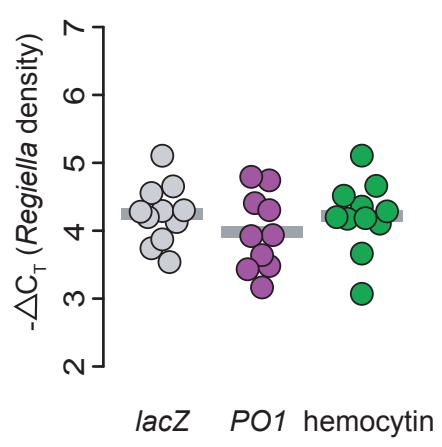

Figure 2: RNAi knockdowns and Regiella density. A: Validation of the RNAi knockdown for $P O 1$ in $1^{\text {st }}$ instar aphids. The $y$-axis shows $-\Delta C_{T}$ values of expression, which can be interpreted on a $\log _{2}$ scale. Collection time-points (72hrs or adults) and treatment are shown along the bottom of the figure; the two Regiella strains are represented by different colors as shown in the key. Bars show the mean of each treatment group, with biological replicates shown as points. B: Regiella density after knockdown of aphids injected as $1^{\text {st }}$ instars. The y-axis shows $-\Delta C_{\text {T }}$ values of symbiont density, which can be interpreted on a $\log _{2}$ scale. Time-points, treatment, and Regiella strain are indicated as in A. C: Validation of the RNAi knockdown for $P O 1$ in adult aphids. Y-axes are as above, treatment is shown along the bottom of the figures, with lacZ and PO1 dsRNA-injected aphids represented by grey and purple dots, respectively. Grey gars shown mean expression for a treatment. The left panel shows expression of PO1, and the right panel shows expression of the other copy of phenoloxidase in the aphid genotype (PO2). D: Knock-down validation of hemocytin. E: Regiella density after knockdown of aphids injected as adults. Grey, purple, and green points represent different treatments (lacZ, PO1, and hemocytin dsRNA injections, respectively). The y-axis shows Regiella density measured by $-\Delta C_{T}$ values

Immune gene suppression differs across aphid biotypes. As noted above the pea aphid species complex includes multiple reproductively isolated 'biotypes' associated with different species of host plants. We repeated the RNAseq experiment to study the effects of Regiella on gene expression across 
multiple aphid biotypes. We used a genotype from the Lotus corniculatus biotype (663), a genotype from Ononis spinosa (C133), and a genotype from Trifolium pratense (C317). For each aphid genotype, we compared replicate lines that had each been infected with an independent Regiella Clade 2 (.313) infection had not been lost using PCR).
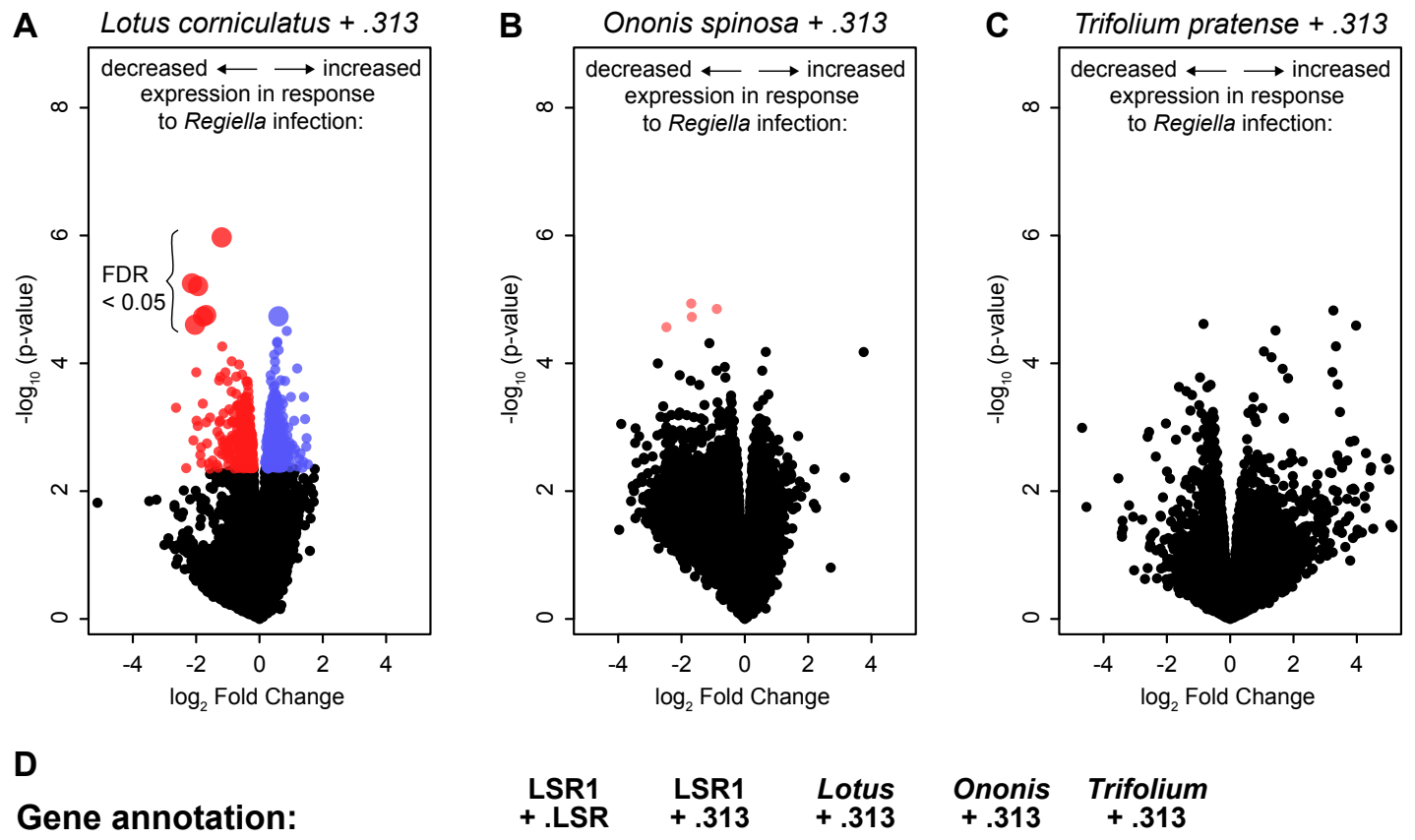

phenoloxidase 2

phenoloxidase 1

plexin A1

hemocytin

apolipoprotein D

uncharacterized protein

uncharacterized protein

plexin A2

eat-4

fibulin-1

uncharacterized protein

EML1

neuronal acetylcholine receptor

toll-like receptor 7

uncharacterized protein

neprilysin

lipid-recognition protein-like

hydroxysteroid dehydrogenase-like SPARC

uncharacterized protein uncharacterized protein uncharacterized protein

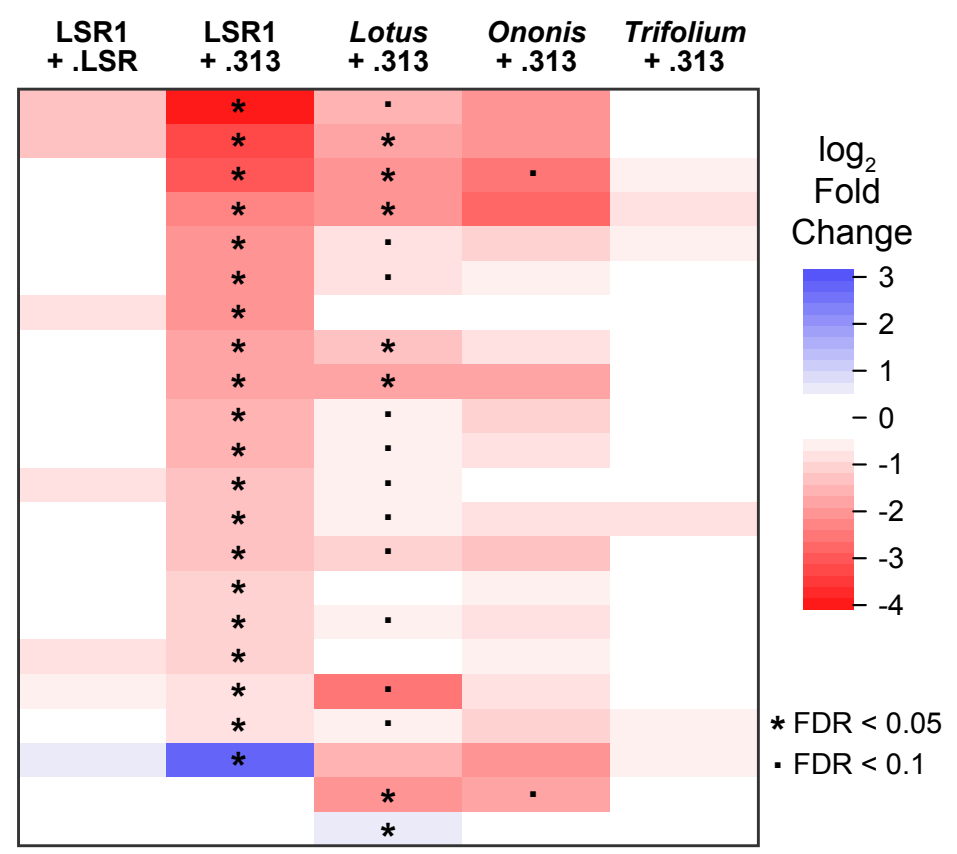

Figure 3: Gene expression across aphid biotypes. A-C: Volcano plots of expression data comparing control vs. Regiella-infected aphids. Each expressed gene in the aphid genome is represented by a point. The $\mathrm{X}$-axes show the $\log _{2}$ fold change of each gene, with points to the right side of each plot indicating increased expression in the presence of symbionts, and points to the left showing decreased expression. The $y$-axes show the - $\log _{10}$ of the $p$-values indicating statistical significance of each gene's expression change. Colored points are those where the expression change was found to be statistically significant at an FDR $<0.1$, with expression at $<0.05$ shown by larger points as indicated. Panels $A, B$, 
and $\mathrm{C}$ show plots for the Lotus, Ononis, and Trifolium genotypes respectively, as shown along the top of the figures. D: A heat-map comparing gene expression in response to Regiella strain .313 infection across host genotypes. The 22 differentially expressed genes identified in the LSR 1 transcriptome, above, are listed to the left of the figure. Colors represent the $\log _{2}$ fold change of these genes in response to Regiella as indicated in the key to the right of the figure (with red panels representing a decrease in expression, and blue indicating an increase in expression). The five transcriptomes generated in this study are shown in each column, as indicated at the top of the figure. Statistical significance of each gene is indicated by * for FDR $<0.05$, and . for FDR $<0.1$.

In aphids from the Lotus corniculatus genotype, harboring Regiella strain .313 had a significant effect on the expression of aphid genes ( 7 genes differentially expressed at an FDR $<0.05$, and 612 genes at an FDR < 0.1; Figure 3A). Of these 7 genes, five were also downregulated by the experiment described above using genotype LSR1, including PO1 and hemocytin (Figure 3D). There is therefore some degree of conservation in the response to Regiella across genetically distinct aphid lines (Figure 3D). In contrast, zero genes were differentially expressed in response to Regiella in the Ononis biotype line at an FDR of $<0.05$, and only 4 genes were differentially expressed at $<0.1$ (Figure 3B). Similarly, zero genes differed in expression in response to Regiella from the Trifolium line (Figure 3C) at either significance level.

\section{F1 genotypes have an intermediate phenotype in symbiont density and immune suppression. We} performed an F1 cross (Figure 4A) between two of the biotype lines in order to better understand the role of host genetic variation in Regiella density and immune gene expression. We crossed the Lotus (663) and Trifolium (C317) lines (52), and generated multiple replicate infections with Regiella strain .313 in each line as above. After four generations, we measured Regiella density using qPCR. Regiella density differed between the parental lines (Figure 4B; post-hoc tests Table S3), with the Lotus line harboring a significantly higher density of Regiella strain .313 (5.2X higher) than the Trifolium line. Further, the F1 lines harbored Regiella at densities intermediate to the parental lines (Figure 4B; post-hoc tests, Table S3).

We then sampled aphids from this same generation to compare changes in immune gene expression due to Regiella in parental and F1 lines. We selected two F1 lines for this assay with each aphid genotype serving as the maternal line. We used qPCR to measure expression of both copies of phenoloxidase, hemocytin, and also nitric oxide synthase (NOS; an important innate immune mechanism that was not significantly differentially expressed in any of our RNAseq studies). Confirming our RNAseq findings, harboring Regiella led to a decrease in expression of PO1, PO2, and Hemocytin in the Lotus genotype, but Regiella did not change gene expression in the Trifolium genotype (Figure 3C). Further, the $\mathrm{F} 1$ lines showed significant differences in gene expression that were intermediate to the two parental lines: three immune genes were suppressed in response to Regiella, but to a significantly lesser extent than in the lotus genotype (Figure 3C; post-hoc tests, Table S4). 
A
Lotus
cornitulatus

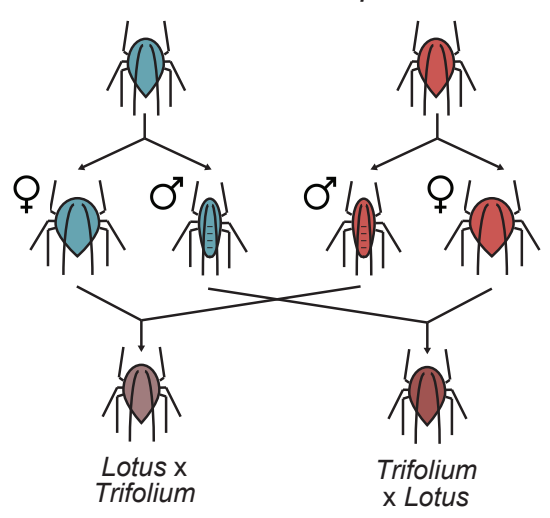

B

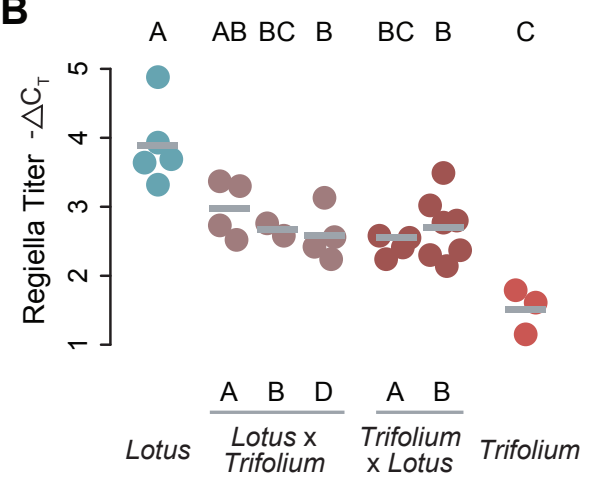

C

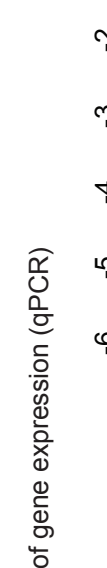

Phenoloxidase 1 (ACYP1004484)

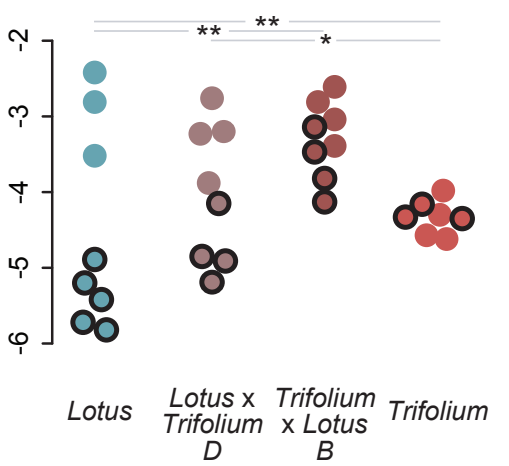

\begin{tabular}{ll} 
& No symbiont \\
Hemocytin & O with Regiella \\
\cline { 2 - 3 }
\end{tabular} (ACYPI003478)

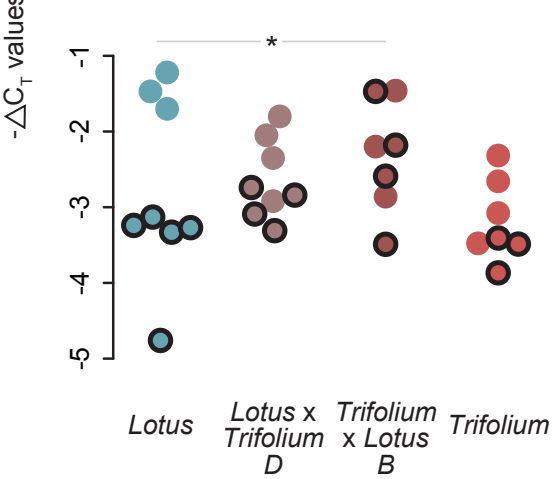

Phenoloxidase 2 (ACYPIO72244)

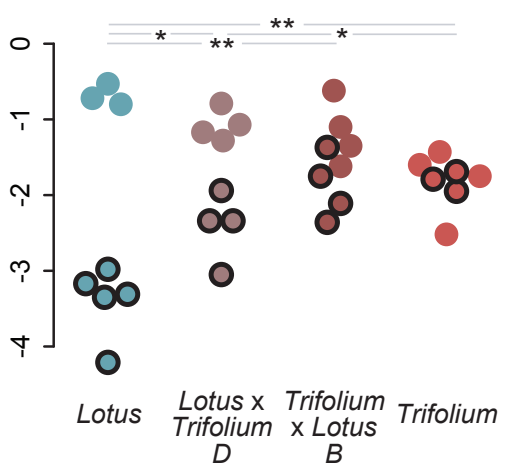

Nitric Oxide Synthase (ACYPI001689)

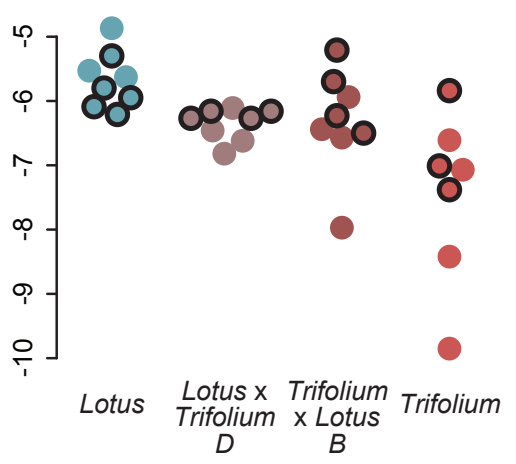

Figure 4: Immune suppression in F1 hybrid lines. A: Diagram of the crossing scheme. B: Regiella density in parental and $\mathrm{F} 1$ lines. The $\mathrm{y}$-axis shows the $-\Delta \mathrm{C}_{\mathrm{T}}$ values reflecting Regiella density, which can be interpreted on a $\log _{2}$ scale. The genotypes are shown along the bottom of the figure. Each biological replicate, representing an independently injected aphid + Regiella line, is shown by a colored point, with the means for each genotype shown in grey bars. Significance groups are shown along the top of the figure at $p<0.05$. C: Immune gene expression in lines with and without Regiella. The y-axis of each plot shows $-\Delta C_{T}$ values (qPCR output) of gene expression. Aphid genotype is shown along the bottom of each figure. Each biological replicate (an independently injected line) is shown by a colored point, with lines harboring Regiella indicated by a dark black outline as indicated in the legend. The results of a post-hoc analysis of the interaction term between Regiella presence/absence and host genotype is indicated at the top of the figure, with an * or ** indicating that two genotypes differ in the extent to which Regiella altered gene expression at $p<0.05$ and $p<0.01$, respectively. 
We show that some aphids harboring the facultative bacterial symbiont Regiella insecticola suppress expression of key immune genes, and we link immune suppression with increases in Regiella density. We further find that this mechanism is influenced by host genetic factors: some genotypes harbor Regiella at lower densities and do not experience altered gene expression when infected, where other aphid genotypes experience suppressed immune gene expression and have higher-density Regiella infections. This study shows that intraspecific variation in the immune system affects a heritable symbiosis.

We found no significantly differentially expressed genes in the transcriptome of aphids harboring a Clade 1 (.LSR) Regiella strain. Using qPCR we confirmed that the immune suppression we uncover occurs more strongly in aphids harboring the Clade 2 Regiella than the Clade 1 strain. An important question is whether symbionts are suppressing host immune mechanisms in order to reach higher densities in hosts, or if hosts are modifying immune mechanisms in order to accommodate symbionts. Fitness costs to aphids of harboring symbionts (including Regiella) have been measured in the laboratory and field (34, 53), and we have found previously that higher density Clade 2 Regiella strains impose stronger survival costs on hosts than the lower density Clade 1 strains (45). In addition, the two Regiella clades confer protection against specific genotypes of the fungal pathogen Pandora neoaphidis (44), and therefore Regiella density will be positively correlated with symbiont-mediated protection for some fungal genotypes and negatively correlated for others. Together these results suggest that immune suppression is not an adaptation on the part of the host in order to accommodate symbionts, but instead some Regiella strains suppress immune mechanisms in order to establish at higher densities in hosts. Establishing at a higher density could benefit Regiella through improved competitive outcomes with other strains and species of symbionts, or through increased horizontal transmission which occurs on evolutionary (43) and even ecological (54) timescales.

Aphid biotypes harbor facultative symbionts at different frequencies that are to some degree conserved across continents (29-32), and decades of research have gone into explaining these patterns in order to better understand the ecological and evolutionary forces shaping beneficial host-microbe interactions. One particularly clear association is between aphids from the Trifolium biotype and Regiella-and specifically Regiella from Clade 2 (43). Studies attempting to explain this pattern have considered factors like the potential effects of Regiella on host plant use (36-39) and pressure from fungal pathogens on different host plants (40). We suggest that host genetic effects represent an additional factor shaping the aphid-symbiont frequencies in natural populations. Whether a cause or consequence of the strong 
aphids are better adapted to harboring this symbiont than biotypes that rarely interact with Clade 2

Regiella. We found no evidence of immune suppression in an aphid genotype from the Trifolium biotype: zero genes were differentially expressed in response to harboring Regiella strain .313 (which was confirmed using qPCR on three immune genes). Together, these results are consistent with a scenario where Clade 2 Regiella have evolved increased within-host density that harms host survival, and aphids from the Trifolium biotype have adapted to prevent immune suppression to control symbiont numbers.

The phenoloxidase enzyme is required for the activation of melanogenesis in invertebrates. Against multicellular parasites, melanin is deposited around a foreign object via immune cells (hemocytes), and the melanin capsule prevents the growth and reproduction of parasites (55). $P O$ is also upregulated in response to microbial pathogens in many studies $(56,57)$ and it is thought that because phenoloxidase is cytotoxic it helps immune cells kill phagocytosed microbes (58). Aphid immune cells express phenoloxidase (59), are known to have phagocytic properties $(59,60)$, and have been shown using microscopy to contain secondary symbionts including Regiella (60). Harboring Regiella (and Hamiltonella but not other species of symbionts) leads to a sharp decrease in the numbers of circulating immune cells (called granulocytes) (60). One possibility is that $P O$ knockdowns via RNAi in our study disrupted the cellular immune responses aphids use to regulate symbionts during development, but the natural mechanisms Regiella are using to suppress $P O$ and other immune genes are unknown.

In addition to the effects on symbionts we have uncovered in this study, phenoloxidase has been shown to be an important part of the pea aphid's response to pathogens (e.g. fungal pathogens $(61,62)$ ). Functionally, a recent study found that silencing of $P O 1$ and PO2 via RNAi leads to decreased resistance of pea aphids against pathogenic bacteria and a generalist fungal pathogen (63). Together, these results show that the same molecular mechanisms are influencing interactions with both beneficial and pathogenic microbes in this system. The protection against specialist fungal pathogens conferred by Regiella might benefit hosts, but immune suppression by some strains of Regiella could trade-off with an increased risk of infection with other pathogens. Recent work on animal immune systems has emphasized the role of immune mechanisms in regulating mutualistic interactions between microbes and hosts. In general, how immune systems evolve to manage the complex task of interacting with distinct microbes with different effects on host fitness is an important question.

Finally, our findings emphasize the importance of host genetic variation in associations with beneficial microbes (64). We found that hybrids between aphid biotypes harbor symbionts at intermediate densities to their parental lines and only partially suppress immune gene expression. The extent to which a host responds to symbiont infection is therefore likely be a quantitative trait, much like resistance against 
selection on the relative costs and benefits of symbiosis. This work thus contributes to a growing view of animal microbiomes as complex phenotypes that influence animal fitness, and that are to some extent under host control.

METHODS

Pea aphids and symbiont establishment: Pea aphids reproduce parthenogenetically under certain light and temperature conditions $\left(16 \mathrm{~L}: 8 \mathrm{D}\right.$ at $\left.20^{\circ} \mathrm{C}\right)$, allowing us to rear large numbers of genetically identical and developmentally synchronized individuals for use in experiments. Wild-collected lines were cured of their original symbiont infections using antibiotics (36) and maintained asexually in the lab for several years before use in experiments (Tables S5 and S6 specify collection information for aphid genotypes and symbiont strains).

Throughout these experiments we used established protocols to infect aphids with facultative symbionts (65-67). We inject a small volume of hemolymph from an infected donor aphid into a $1^{\text {st }}$ instar recipient using a glass capillary needle. We then rear these aphids to adulthood and then collect an offspring from late in the birth-order to establish the infected line. When this aphid produces offspring, we extract DNA (using a lysis buffer with proteinase $\mathrm{K}$ and an ethanol precipitation (68)) and screen the line for symbionts using PCR with symbiont-specific primers (43) (Table S7): $\left(94^{\circ} \mathrm{C} 2 \mathrm{~min}, 11\right.$ cycles of $94^{\circ} \mathrm{C}$ $20 \mathrm{~s}, 56^{\circ} \mathrm{C}$ (declining $1^{\circ} \mathrm{C}$ each cycle) $50 \mathrm{~s}, 72^{\circ} \mathrm{C} 30 \mathrm{~s}, 25$ cycles of $94^{\circ} \mathrm{C} 2 \mathrm{~min}, 45^{\circ} \mathrm{C} 50 \mathrm{~s}, 72^{\circ} \mathrm{C} 2 \mathrm{~min}$ and a final extension of $72^{\circ} \mathrm{C} 5 \mathrm{~min}$ ). Each biological replicate in these experiments (an "aphid line") originated from a separate symbiont-injection and screening except where noted.

Measurements of symbiont density using qPCR: We established aphid lines from the LSR1 genotype 399 with two strains of Regiella: "Clade 1" Regiella (strain .LSR), and "Clade 2" Regiella (strain .313). These two strains of Regiella each come from one of the two main clades of Regiella found among pea aphids as determined using an established protocol for MLST sequence typing $(44,69)$. We reared lines that had successfully acquired a Regiella infection under asexual conditions for four generations, at which point we re-screened lines for Regiella infection. We then used qPCR to compare symbiont density between these strains. We removed embryos from groups of 7 adult aphids, and extracted DNA using the Qiagen DNEasy kit under recommended conditions. We used qPCR primers that amplify a conserved region of the Regiella $h r p A$ gene (Table S7). Amplification of $g 3 P D H$ was used as an endogenous reference gene that controlled for the relative abundance of host DNA in each sample. Primer concentrations were optimized against a serial dilution of gDNA (400/350nM F/R and 300nM for 
values with a t-test. Note that this approach reveals the relative density of symbionts relative to host tissue across different samples, but does not measure the absolute abundance of symbionts.

413 Effects of Regiella on host gene expression using RNAseq: We then measured the effects of 414 harboring Regiella on gene expression using RNAseq. We used the lines established above with either 415 Regiella strain .LSR or .313, and symbiont-free aphids of the same host genotype. For the 'no symbiont' 416 treatment, we sham injected aphids (injected aphids with a small volume of hemolymph $(0.25 \mu \mathrm{l})$ from an 417 uninfected adult donor aphid) and handled aphids in the same way as with symbiont-injected aphids.

418 For transcriptome sequencing, we collected adult, fourth generation aphids on the first day that each 419 line produced offspring and dissected and removed developing embryos (in order to measure gene 420 expression of the mother without including RNA from her embryos). We stored carcasses in TRIzol 421 (Invitrogen) at $-80^{\circ} \mathrm{C}$. Each sample contained $\sim 14$ adult carcasses collected from multiple host plants. 422 We extracted RNA using TRIzol-chloroform and an isopropanol precipitation with an ethanol wash. We 423 digested genomic DNA and cleaned the RNA using the Zymo Clean \& Concentrate-5 kit with the DNAse 424 I enzyme. RNA quality control was conducted on a bioanalyzer chip, and 12 sequencing libraries (4 425 biological replicates $\times 3$ treatments) were constructed using the NEBNext Ultra II RNA Library Prep Kit 426 for Illumina (including poly-A selection and 15 rounds of PCR amplification). Libraries were sequenced across one lane of Illumina PE150 sequencing (approximately 20 million reads per library) with a 250$300 b p$ insert per library.

429

430 RNAseq analysis: We estimated the average insert size of paired-end libraries using Picard Tools 431 v.2.21.3 in java 1.8.0, and mapped reads to the pea aphid reference genome v.2.1 (42) using tophat 432 v.2.1.1 (70). We counted reads mapped to each annotated gene (using a modified version of pea aphid genome annotation v.2.1 (https://bipaa.genouest.org/sp/acyrthosiphon_pisum/) with several duplicated genes removed from the file) using the count function in htseq v.0.9.1 (71) and the 'union' overlap mode (Table S8). We analyzed read counts using EdgeR v.3.22.3 in R v.3.5.0. Genes with a minimum threshold of aligned reads, determined by the filterByExpr function in edgeR, were retained in the analysis. We fit a quasi-likelihood model to the data using the glmQLFit function, and we tested for statistically significant differential expression of each gene using a quasi-likelihood F-test, interpreting genes with a false discovery rate (FDR) of $<0.05$ as differentially expressed in response to Regiella infection.

442 Immune gene expression across facultative symbiont species via qPCR: We used qPCR to verify 443 our RNAseq results, and to explore how differences in gene expression due to Regiella in key innate 444 immunity genes varied across facultative symbiont species. We used qPCR primers (Table S7) that 445 amplified 80-120bp of two target genes of interest (PO1: ACYPI004484 and hemocytin: ACYPI003478) 
446 and four endogenous control genes (Glyceraldehyde 3-phosphate dehydrogenase ( $g 3 P D H)$ :

447 ACYPI009769, NADH dehydrogenase: ACYPI009382, $\beta$-tubulin: ACYPI001007, and Rp/32:

448 ACYPI000074). Primer concentrations were optimized against a 1:10 serial dilution of gDNA (200ng -

$449 \quad 0.2 n g$ gDNA per reaction) to an efficiency of 100 +/- 10\% (PO1: 100nM; hemocytin: 100nM; $g 3 P D H$ :

450 400/350nM F/R; NADH: 350/300nM F/R; $\beta$-tubulin: 400nM; and rp/32: 200nM). Reactions were run on a

451 Bio-RAD CFX96 Real-Time System machine, with an initial step of $95^{\circ} \mathrm{C}$ for 3 minutes and 40 cycles of $45295^{\circ} \mathrm{C}$ for $10 \mathrm{~s}$ and $60^{\circ} \mathrm{C}$ for 30 s. Each $20 \mu \mathrm{L}$ reaction included a $1 \mathrm{X}$ PCR buffer, $\mathrm{Mg}^{+2}$ at $2 \mathrm{mM}$, dNTPs at $4530.2 \mathrm{mM}$, EvaGreen at $1 \mathrm{X}, 0.025$ units $/ \mu \mathrm{L}$ of Invitrogen taq, and $40 \mathrm{ng}$ of cDNA. Three technical replicates 454 were run for each reaction.

455 We measured expression of these genes in lines with and without symbionts in two separate 456 experiments. First, we collected aphids from the Regiella-infected lines used for the RNAseq above (no 457 symbionts, Clade 1 .LSR Regiella, and Clade 2.313 Regiella). We dissected out and removed embryos, 458 pooled adult carcasses, extracted and cleaned RNA, and DNAse treated samples as above. We 459 synthesized cDNA using the BioRad iScript cDNA synthesis kit under recommended conditions. For 460 each sample we averaged the $\mathrm{C}_{\mathrm{T}}$ values from the endogenous control genes, and calculated $-\Delta \mathrm{C}_{\mathrm{T}}$ values 461 by - $\left(C_{T \text { target }}-C_{T \text { mean endogenous control }}\right)$. We analyzed differences in gene expression between symbiont-free, 462 clade 1, and clade 2 lines with one-way ANOVAs on the $-\Delta \mathrm{C}_{\mathrm{T}}$ values, and used Tukey HSD tests for pair463 wise comparisons among different symbiont backgrounds. We performed separate analyses for the two 464 genes.

465 In a second experiment, we injected three additional symbiont species into aphids and measured $466 P 01$ and hemocytin expression as above. For donor aphids, we used an aphid line harboring Serratia 467 symbiotica, a line harboring Spiroplasma sp. (strain .161), and two strains of Hamiltonella defensa (Table 468 S6). We only successfully established Serratia from two injection events after multiple attempts, and so 469 the biological replicates of this assay were generated by splitting the lines onto multiple plants after 2 470 generations before sampling at generation 5; the other lines represent independently injected lines. We 471 maintained sham-injected (symbiont-free) aphids under identical conditions as above. Gene expression 472 was measured and analyzed as above. We note that the Spiroplasma sp. strain used in this experiment 473 has been shown, like Regiella, to be protective against fungal pathogens while the other symbiont 474 species used have not found to confer fungal protection. The two Hamiltonella strains used were 475 collected in the same field and may not represent distinct symbiont genotypes from each other. Data 476 were analyzed as above.

477

478 Expression knock-down via RNAi: We designed primers that amplify regions of two target genes 479 (531bp of PO1 and 483bp of Hemocytin) with the T7 promoter sequence 480 (TAATACGACTCACTATAGGG) on the 5' end of each primer using the e-RNAi Webservice 481 (https://www.dkfz.de/signaling/e-rnai3/). Primer sequences can be found in Table S7. We PCR amplified 
these regions from cDNA under recommended conditions. PCR products were sequenced using Sanger sequencing primed with the T7 promoter sequence to confirm target identity. We then purified $160 \mu \mathrm{L}$ of PCR product (using NaOAc and EtOH precipitation) and concentrated it to $500 \mathrm{ng} / \mu \mathrm{L}$. We used the MEGAScript RNAi kit to synthesize dsRNA from PCR amplicons under recommended conditions and a $15 \mathrm{hr}$ transcription incubation. We ran dsRNA (at a 1/400 dilution) on a $2 \%$ agarose gel to verify that a single band was obtained of the correct size, and we then concentrated the dsRNA product to approximately $3300 \mathrm{ng} / \mu \mathrm{L}$ using $\mathrm{LiCl}$ and an ethanol precipitation, and eluted the final dsRNA product in MEGAScript buffer. We repeated these protocols to generate dsRNA from lacZ as a control as in (72).

In a first experiment, we injected $1^{\text {st }}$ instar (1-day-old) aphids with approximately $100 \mu \mathrm{g}$ dsRNA from either PO1 or lacZ as a control using a glass capillary needle attached to a syringe on the underside of thorax of each aphid. We used two aphid lines that harbored either Regiella strain .LSR or .313. We collected injected aphids at two time-points after injection: at $72 \mathrm{hrs}$ and at day 9 when they had undergone their final molt to the adult stage. For the $72 \mathrm{hr}$ samples, groups of 3 whole aphids were pooled in TRIzol and stored at $-80^{\circ} \mathrm{C}$ for RNA extraction, or were stored in tubes at $-20^{\circ} \mathrm{C}$ for gDNA extraction. For the adult samples, we dissected out developing embryos and stored adult carcasses individually for RNA or DNA extraction, and we also pooled embryos from three adults and stored them in tubes for DNA extraction.

From the samples stored in TRIzol, we extracted RNA, synthesized cDNA, and measured the expression of $P O 1$ and four endogenous control genes using qPCR as above. We calculated $-\Delta \mathrm{C}_{\mathrm{T}}$ values as above and analyzed these using two-way ANOVAs (with treatment (lacZ vs. PO1) and Regiella strain (.LSR vs. .313) as factors). We conducted post-hoc analyses using Tukey's HSD tests. The two time-points were analyzed separately. We extracted DNA from the remaining samples using the Qiagen DNEasy kit and measured Regiella densities using qPCR amplification of the hrpA gene as above. We analyzed these data using two-way ANOVAs and Tukey's HSD tests as above in R v.3.5.1 after testing for model assumptions.

For adult injections, we reared LSR1 aphids with Regiella strains .LSR and .313 at low densities as 508 509 above. We then injected adult aphids (9 days old) with $0.3 \mu \mathrm{L}$ of dsRNA (approximately $1 \mu \mathrm{g}$ total). In this experiment we performed knock-downs of two genes: PO1 and Hemocytin. We first collected aphids harboring strain .LSR at 72 hours after injection to validate our knock-downs. For each gene, we pooled groups of three aphids into 8 samples, removed developing embryos, extracted RNA from adult carcasses, synthesized cDNA, and measured gene expression as above. We analyzed $-\Delta \mathrm{C}_{\mathrm{T}}$ values for each gene using t-tests with treatment as the independent variable. In this experiment we also measured expression of $P O 2$ in aphids that had been injected with dsRNA from PO1 to verify that our knock-down was specific to PO1. We then injected adult aphids harboring either strain .LSR or strain .313 with approximately $1 \mu \mathrm{g}$ dsRNA, removed and discarded developing embryos after $72 \mathrm{hrs}$, pooled samples into groups of 4 dissected aphids, extracted DNA as above, and measured Regiella densities using qPCR as 
above. We analyzed $-\Delta \mathrm{C}_{\mathrm{T}}$ values from this experiment using a two-way ANOVA, and included symbiont strain (.LSR or .313) and treatment (lacZ (control), PO1, and Hemocytin) as factors in the analysis. We conducted post-hoc analyses using a Tukey's HSD test to compare levels within treatment.

RNAseq on aphid genotypes from multiple host-plant associated biotypes: We selected three

aphid genotypes each from a different host-plant associated biotype (Lotus corniculatus, Ononis spinosa, and Trifolium pratense); information on collection location can be found in Table S5. We established Regiella strain .313 infections in each line as above. Each Regiella-infected line was established from a different symbiont injection and maintained separately on $V$. faba plants. In parallel, control aphids were sham injected as above. After 4 generations, we froze seven adult aphids from each line in liquid nitrogen and stored at $-80^{\circ} \mathrm{C}$. RNA was extracted and purified as above. For the Trifolium pratense biotype, RNAseq libraries and sequencing was conducted as described for the LSR genotype used above. For the Lotus corniculatus and Ononis spinosa biotypes, dual-indexed stranded sequencing libraries were constructed using the NEBNext polyA selection and Ultra Directional RNA library preparation kits. Libraries were sequenced on one lane of Illumina HiSeq 4000 (Paired-end 150bp) generating a target of $>2 \times 280 \mathrm{M}$ reads. We analyzed each genotype separately, comparing libraries with and without a Regiella infection, as above.

F1 crosses: We used two of these biotype lines (C317 from Trifolium and 663 from Lotus) for the F1 genetic cross because we found from RNAseq data that these lines responded differently to Regiella infection. To induce male and female aphids for genetic crosses, we transferred stocks to 'autumn' conditions (short day, $13 \mathrm{~L}: 11 \mathrm{D}$ at $18^{\circ} \mathrm{C}$ ). After 30 days we moved third and fourth instar nymphs onto leaf-plates (a fava bean leaf in $2 \%$ agar in Petri dishes) to isolate virgin egg-laying sexual females (oviparae) and males. Oviparae have a characteristic thicker hind tibia, and this feature was used to isolate probable oviparae from males. The male screening was less stringent because virgin males were not needed. We setup each cross by placing the corresponding genotype oviparae and males onto a fava bean seedling, replenishing breeding stocks as they became available. After 24 hours, we treated melanized eggs with $10 \%$ calcium propionate to clean off the surface and then transferred eggs using fine-tipped forceps to a small petri dish with Whatman filter paper moistened with sterile water. We sealed the plates with parafilm wrap and left them in autumn conditions for a further 24 hours, after which dishes were transferred to a $2^{\circ} \mathrm{C}$ incubator to diapause. After 3 months, eggs were removed from the diapause conditions and with fine-tip forceps, rolled against a Kimwipe to reduce any microbial growth. We then transferred diapaused eggs to a new leaf-plate and placed them in 'autumn' conditions (as above) until a fundatrix hatched. Each fundatrix was separated and a line was considered stable after two generations. We used this protocol to generate five F1 lines: three with line 663 as the maternal 
qPCR measures of gene expression in the F1 panel: We established replicate Regiella strain .313

We then measured expression of four immune genes aphids with and without Regiella using qPCR in 562 the parental and two of the $\mathrm{F} 1$ lines. Four generations after injection, four aphids from each biological replicate from each genotype were removed from plants, embryos were dissected out of adult carcasses and stored in TRIzol at -80C. We extracted RNA and synthesized cDNA as above. For each sample, we 565 measured expression against the four endogenous control genes used above. Here we measured expression of four target genes: two copies of Phenoloxidase (PO1: ACYPI04484 and PO2: ACYPI072244), Hemocytin (ACYPI003478), and Nitric Oxide Synthase (ACYPI001689). $-\Delta C_{T}$ values were analyzed using an ANOVA after testing for model assumptions; post-hoc tests using the multcomp package in $\mathrm{R}$ v.3.5.0 were conducted to compare the interaction terms between Regiella presence/absence and host genotype. Analysis of expression of each gene was conducted separately.

ACKNOWLedgementS: BJP is a Pew Scholar in the Biomedical Sciences, supported by The Pew Charitable Trusts. OSZ is supported by an NSF Graduate Research Fellowship.

574

STATEMENT OF AUTHORSHIP: HLN, EBG, OSZ, and BJP carried out the molecular and experimental work. BJP conceived of the study, analyzed the data, wrote the manuscript, and funded the work. All authors edited and approved the manuscript before submission. 


\section{REFERENCES}

1. K. Hilgenboecker, P. Hammerstein, P. Schlattmann, A. Telschow, J. H. Werren, How many species are infected with Wolbachia?--A statistical analysis of current data. FEMS Microbiol Lett 281, 215-220 (2008).

2. N. A. Moran, J. P. McCutcheon, A. Nakabachi, Genomics and evolution of heritable bacterial symbionts. Annu Rev Genet 42, 165-190 (2008).

3. J. Ferrari, F. Vavre, Bacterial symbionts in insects or the story of communities affecting communities. Philos Trans R Soc Lond B Biol Sci 366, 1389-1400 (2011).

4. H. Hawlena et al., The arthropod, but not the vertebrate host or its environment, dictates bacterial community composition of fleas and ticks. ISME J 7, 221-223 (2013).

5. J. A. Chandler, J. M. Lang, S. Bhatnagar, J. A. Eisen, A. Kopp, Bacterial communities of diverse Drosophila species: ecological context of a host-microbe model system. PLoS Genet 7, e1002272 (2011).

6. A. H. C. McLean, H. C. J. Godfray, J. Ellers, L. M. Henry, Host relatedness influences the composition of aphid microbiomes. Environmental Microbiology Reports 11, 808-816 (2019).

7. J. Jaenike, R. Unckless, S. N. Cockburn, L. M. Boelio, S. J. Perlman, Adaptation via symbiosis: recent spread of a Drosophila defensive symbiont. Science 329, 212-215 (2010).

8. A. K. Hansen, G. Jeong, T. D. Paine, R. Stouthamer, Frequency of secondary symbiont infection in an invasive psyllid relates to parasitism pressure on a geographic scale in California. Appl Environ Microbiol 73, 7531-7535 (2007).

9. R. A. Chong, N. A. Moran, Intraspecific genetic variation in hosts affects regulation of obligate heritable symbionts. Proc Natl Acad Sci U S A 113, 13114-13119 (2016).

10. L. J. Funkhouser-Jones, E. J. van Opstal, A. Sharma, S. R. Bordenstein, The Maternal Effect Gene Wds Controls Wolbachia Titer in Nasonia. Curr Biol 28, 1692-1702 e1696 (2018).

11. K. S. Stoy, A. K. Gibson, N. M. Gerardo, L. T. Morran, A need to consider the evolutionary genetics of host-symbiont mutualisms. J Evol Biol 10.1111/jeb.13715 (2020).

12. F. H. Login et al., Antimicrobial peptides keep insect endosymbionts under control. Science 334, 362-365 (2011).

13. J. Maire, C. Vincent-Monegat, F. Masson, A. Zaidman-Remy, A. Heddi, An IMD-like pathway mediates both endosymbiont control and host immunity in the cereal weevil Sitophilus spp.

Microbiome 6, 6 (2018).

14. J. K. Herren, B. Lemaitre, Spiroplasma and host immunity: activation of humoral immune responses increases endosymbiont load and susceptibility to certain Gram-negative bacterial pathogens in Drosophila melanogaster. Cell Microbiol 13, 1385-1396 (2011).

15. X. Pan et al., The bacterium Wolbachia exploits host innate immunity to establish a symbiotic relationship with the dengue vector mosquito Aedes aegypti. ISME J 12, 277-288 (2018).

16. R. V. Rio, Y. N. Wu, G. Filardo, S. Aksoy, Dynamics of multiple symbiont density regulation during host development: tsetse fly and its microbial flora. Proc Biol Sci 273, 805-814 (2006).

17. A. J. Shultz, T. B. Sackton, Immune genes are hotspots of shared positive selection across birds and mammals. Elife 8 (2019).

18. T. A. Schlenke, D. J. Begun, Natural selection drives Drosophila immune system evolution. Genetics 164, 1471-1480 (2003).

19. C. Drosophila 12 Genomes et al., Evolution of genes and genomes on the Drosophila phylogeny. Nature 450, 203-218 (2007).

20. B. P. Lazzaro, T. J. Little, Immunity in a variable world. Philos Trans R Soc Lond B Biol Sci 364, 15-26 (2009).

21. J. Rolff, M. T. Siva-Jothy, Invertebrate ecological immunology. Science 301, 472-475 (2003).

22. R. L. Unckless, B. P. Lazzaro, The potential for adaptive maintenance of diversity in insect antimicrobial peptides. Philos Trans R Soc Lond B Biol Sci 371 (2016).

23. J. C. Brownlie, K. N. Johnson, Symbiont-mediated protection in insect hosts. Trends Microbio/ 17, 348-354 (2009).

24. J. Martinez et al., Should Symbionts Be Nice or Selfish? Antiviral Effects of Wolbachia Are Costly but Reproductive Parasitism Is Not. PLoS Pathog 11, e1005021 (2015). 
25. K. M. Oliver, J. Campos, N. A. Moran, M. S. Hunter, Population dynamics of defensive symbionts in aphids. Proc Biol Sci 275, 293-299 (2008).

26. J. A. Brisson, D. L. Stern, The pea aphid, Acyrthosiphon pisum: an emerging genomic model system for ecological, developmental and evolutionary studies. Bioessays 28, 747-755 (2006).

27. V. Fazalova, B. Nevado, Low Spontaneous Mutation Rate and Pleistocene Radiation of Pea Aphids. Mol Biol Evol 37, 2045-2051 (2020).

28. J. Peccoud, A. Ollivier, M. Plantegenest, J. C. Simon, A continuum of genetic divergence from sympatric host races to species in the pea aphid complex. Proc Natl Acad Sci U S A 106, 74957500 (2009).

29. J. Ferrari, J. A. West, S. Via, H. C. Godfray, Population genetic structure and secondary symbionts in host-associated populations of the pea aphid complex. Evolution 66, 375-390 (2012).

30. S. H. Chung, B. J. Parker, F. Blow, J. A. Brisson, A. E. Douglas, Host and symbiont genetic determinants of nutritional phenotype in a natural population of the pea aphid. Mol Ecol 10.1111/mec.15355 (2020).

31. J. A. Russell et al., Uncovering symbiont-driven genetic diversity across North American pea aphids. Mol Ecol 22, 2045-2059 (2013).

32. T. Tsuchida, R. Koga, H. Shibao, T. Matsumoto, T. Fukatsu, Diversity and geographic distribution of secondary endosymbiotic bacteria in natural populations of the pea aphid, Acyrthosiphon pisum. Mol Ecol 11, 2123-2135 (2002).

33. C. L. Scarborough, J. Ferrari, H. C. Godfray, Aphid protected from pathogen by endosymbiont. Science 310, 1781 (2005).

34. B. J. Parker, C. J. Spragg, B. Altincicek, N. M. Gerardo, Symbiont-mediated protection against fungal pathogens in pea aphids: a role for pathogen specificity? Appl Environ Microbiol 79, 24552458 (2013).

35. P. Lukasik, M. van Asch, H. Guo, J. Ferrari, H. C. Godfray, Unrelated facultative endosymbionts protect aphids against a fungal pathogen. Ecol Lett 16, 214-218 (2013).

36. A. H. McLean, M. van Asch, J. Ferrari, H. C. Godfray, Effects of bacterial secondary symbionts on host plant use in pea aphids. Proc Biol Sci 278, 760-766 (2011).

37. J. Ferrari, C. L. Scarborough, H. C. Godfray, Genetic variation in the effect of a facultative symbiont on host-plant use by pea aphids. Oecologia 153, 323-329 (2007).

38. T. E. Leonardo, Removal of a specialization-associated symbiont does not affect aphid fitness. Ecology Letters 7, 461-468 (2004).

39. T. Tsuchida, R. Koga, T. Fukatsu, Host plant specialization governed by facultative symbiont. Science 303, 1989 (2004).

40. J. Hrcek et al., Hosts do not simply outsource pathogen resistance to protective symbionts. Evolution 10.1111/evo.13512 (2018).

41. H. Mathe-Hubert, H. Kaech, C. Hertaeg, J. Jaenike, C. Vorburger, Nonrandom associations of maternally transmitted symbionts in insects: The roles of drift versus biased cotransmission and selection. Mol Ecol 28, 5330-5346 (2019).

42. C. International Aphid Genomics, Genome sequence of the pea aphid Acyrthosiphon pisum. PLoS Biol 8, e1000313 (2010).

43. L. M. Henry et al., Horizontally transmitted symbionts and host colonization of ecological niches. Curr Biol 23, 1713-1717 (2013).

44. B. J. Parker, J. Hrcek, A. H. C. McLean, H. C. J. Godfray, Genotype specificity among hosts, pathogens, and beneficial microbes influences the strength of symbiont-mediated protection. Evolution 71, 1222-1231 (2017).

45. B. J. Parker, J. Hrcek, A. H. C. McLean, J. A. Brisson, H. C. J. Godfray, Intraspecific variation and within-host density in an insect-microbe symbiosis. bioRxiv 10.1101/2020.11.03.365353 (2020).

46. I. Arai et al., Immunohistochemical analysis of the role of hemocytin in nodule formation in the larvae of the silkworm, Bombyx mori. J Insect Sci 13, 125 (2013).

47. W. Ni et al., Hemocytin facilitates host immune responses against Nosema bombycis. Dev Comp Immunol 103, 103495 (2020). 
48. A. H. C. McLean et al., Multiple phenotypes conferred by a single insect symbiont are independent. Proc Biol Sci 287, 20200562 (2020).

49. P. Lukasik, H. Guo, M. van Asch, J. Ferrari, H. C. Godfray, Protection against a fungal pathogen conferred by the aphid facultative endosymbionts Rickettsia and Spiroplasma is expressed in multiple host genotypes and species and is not influenced by co-infection with another symbiont. J Evol Biol 26, 2654-2661 (2013).

50. K. M. Oliver, P. H. Degnan, G. R. Burke, N. A. Moran, Facultative symbionts in aphids and the horizontal transfer of ecologically important traits. Annu Rev Entomol 55, 247-266 (2010).

51. C. Ye et al., Induction of RNAi Core Machinery's Gene Expression by Exogenous dsRNA and the Effects of Pre-exposure to dsRNA on the Gene Silencing Efficiency in the Pea Aphid (Acyrthosiphon pisum). Front Physiol 9, 1906 (2018).

52. C. Braendle, M. C. Caillaud, D. L. Stern, Genetic mapping of aphicarus -- a sex-linked locus controlling a wing polymorphism in the pea aphid (Acyrthosiphon pisum). Heredity (Edinb) $\mathbf{9 4}$, 435-442 (2005).

53. J. Hrcek, A. H. McLean, H. C. Godfray, Symbionts modify interactions between insects and natural enemies in the field. J Anim Ecol 85, 1605-1612 (2016).

54. O. Duron, T. E. Wilkes, G. D. Hurst, Interspecific transmission of a male-killing bacterium on an ecological timescale. Ecol Lett 13, 1139-1148 (2010).

55. I. González-Santoyo, A. Córdoba-Aguilar, Phenoloxidase: a key component of the insect immune system. Entomologia Experimentalis et Applicata 142, 1-16 (2012).

56. Y. Wang et al., Activation of Aedes aegypti prophenoloxidase- 3 and its role in the immune response against entomopathogenic fungi. Insect Mol Biol 26, 552-563 (2017).

57. O. Binggeli, C. Neyen, M. Poidevin, B. Lemaitre, Prophenoloxidase activation is required for survival to microbial infections in Drosophila. PLoS Pathog 10, e1004067 (2014).

58. L. Cerenius, B. L. Lee, K. Soderhall, The proPO-system: pros and cons for its role in invertebrate immunity. Trends Immunol 29, 263-271 (2008).

59. A. M. Laughton, J. R. Garcia, B. Altincicek, M. R. Strand, N. M. Gerardo, Characterisation of immune responses in the pea aphid, Acyrthosiphon pisum. J Insect Physiol 57, 830-839 (2011).

60. A. Schmitz et al., The cellular immune response of the pea aphid to foreign intrusion and symbiotic challenge. PLoS One 7, e42114 (2012).

61. B. J. Parker, S. M. Barribeau, A. M. Laughton, L. H. Griffin, N. M. Gerardo, Life-history strategy determines constraints on immune function. J Anim Ecol 86, 473-483 (2017).

62. M. N. Grell, A. B. Jensen, P. B. Olsen, J. Eilenberg, L. Lange, Secretome of fungus-infected aphids documents high pathogen activity and weak host response. Fungal Genet Biol 48, 343352 (2011).

63. L. Xu, L. Ma, W. Wang, L. Li, Z. Lu, Phenoloxidases are required for the pea aphid's defence against bacterial and fungal infection. Insect Mol Biol 28, 176-186 (2019).

64. E. R. Davenport, Elucidating the role of the host genome in shaping microbiome composition. Gut Microbes 7, 178-184 (2016).

65. R. Koga, T. Tsuchida, T. Fukatsu, Changing partners in an obligate symbiosis: a facultative endosymbiont can compensate for loss of the essential endosymbiont Buchnera in an aphid. Proc Biol Sci 270, 2543-2550 (2003).

66. K. M. Oliver, J. A. Russell, N. A. Moran, M. S. Hunter, Facultative bacterial symbionts in aphids confer resistance to parasitic wasps. Proc Natl Acad Sci U S A 100, 1803-1807 (2003).

67. D. Q. Chen, A. H. Purcell, Occurrence and transmission of facultative endosymbionts in aphids. Curr Microbiol 34, 220-225 (1997).

68. W. Bender, P. Spierer, D. S. Hogness, Chromosomal Walking and Jumping to Isolate DNA from the Ace and rosy Loci and the Bithorax Complex in Drosophila melanogaster. J. Mol. Biol. 168, 17-33 (1983).

69. L. M. Henry, M. C. Maiden, J. Ferrari, H. C. Godfray, Insect life history and the evolution of bacterial mutualism. Ecol Lett 18, 516-525 (2015).

70. D. Kim et al., TopHat2: accurate alignment of transcriptomes in the presence of insertions, deletions and gene fusions. Genome Biol 14, R36 (2013). 
741 71. S. Anders, P. T. Pyl, W. Huber, HTSeq--a Python framework to work with high-throughput sequencing data. Bioinformatics 31, 166-169 (2015).

72. B. J. Parker, J. A. Brisson, A Laterally Transferred Viral Gene Modifies Aphid Wing Plasticity. Curr Biol 29, 2098-2103 e2095 (2019).

73. B. Li et al., A large genomic insertion containing a duplicated follistatin gene is linked to the pea aphid male wing dimorphism. eLife 9 (2020). 
749 Table S1: Significantly differentially expressed genes from the RNAseq experiments. Numbers show $\log _{2}$ fold changes of expression in aphids with vs. without Regiella.

\begin{tabular}{|c|c|c|c|c|c|c|}
\hline Gene ID & Annotation & $\begin{array}{l}\text { LSR } \\
+. \text {.LSR }\end{array}$ & $\begin{array}{l}\text { LSR } \\
+.313\end{array}$ & $\begin{array}{l}\text { Lotus } \\
+.313\end{array}$ & $\begin{array}{l}\text { Ononis } \\
+.313\end{array}$ & $\begin{array}{l}\text { Trifolium } \\
+.313\end{array}$ \\
\hline ACYPI061678 & plexin A & 0.03 & -1.79 ** & $-1.19^{* *}$ & -0.86 & -0.41 \\
\hline ACYPI44738 & plexin A1-like † & -0.19 & $-3.09 * *$ & $-2.13^{* *}$ & $-2.48 *$ & -0.46 \\
\hline ACYPI003478 & Hemocytin & -0.37 & -2.24 ** & $-1.94 * *$ & -2.75 & -0.78 \\
\hline ACYPI004484 & Phenoloxidase 1 (subunit A3) & -1.40 & $-3.25^{* *}$ & $-1.68^{* *}$ & -2.06 & -0.14 \\
\hline ACYPI009767 & uncharacterized protein & -- & -- & $0.60 * *$ & 0.16 & -- \\
\hline ACYPI008883 & $\begin{array}{l}\text { probable vesicular glutamate transporter eat- } 4 \\
\dagger\end{array}$ & -0.13 & $-1.89 * *$ & -1.78 ** & -1.71 & -0.05 \\
\hline ACYPI53900 & Uncharacterized protein & -0.34 & 0.09 & -2.04 ** & $-1.67 *$ & -0.45 \\
\hline ACYPI008487 & Apolipoprotein D & 0.03 & $-2.08^{* *}$ & $-0.83 *$ & -1.04 & -0.50 \\
\hline ACYPI006183 & Uncharacterized protein & 0.17 & $-1.92 * *$ & $-0.88 *$ & -0.69 & -0.22 \\
\hline ACYPI072244 & Phenoloxidase 2 (subunit 2) & -1.38 & $-3.87^{* *}$ & $-1.56 *$ & -2.08 & -0.20 \\
\hline ACYPI001483 & $\begin{array}{l}\text { Echinoderm microtubule-associated protein- } \\
\text { like } 1\end{array}$ & -0.76 & -1.25 ** & $-0.49 *$ & -0.45 & -0.18 \\
\hline ACYPI001736 & Uncharacterized protein & 0.09 & -1.09 ** & -0.33 & -0.64 & -0.15 \\
\hline ACYPI50923 & Fibulin-1 † & -0.22 & $-1.52 * *$ & $-0.62 *$ & -1.12 & -0.37 \\
\hline ACYPI009930 & Uncharacterized protein & -0.77 & $-2.13^{* *}$ & 0.09 & -- & -0.12 \\
\hline ACYPI007618 & Neprilysin-11-like † & 0.37 & -1.08 ** & $-0.64 *$ & -0.88 & -0.21 \\
\hline ACYPI007421 & Uncharacterized protein & 0.31 & $-1.44 * *$ & $-0.57^{*}$ & -0.88 & -0.36 \\
\hline ACYPI001380 & $\begin{array}{l}\text { Cys-loop ligand-gated ion channel subunit-like } \\
\text { / Neuronal acetylcholine receptor subunit }\end{array}$ & 0.06 & $-1.28 * *$ & $-0.60 *$ & -0.79 & -0.71 \\
\hline ACYPI061541 & Uncharacterized protein & 0.74 & $2.71^{* *}$ & -1.63 & -2.10 & -0.51 \\
\hline ACYPI001359 & $\begin{array}{l}\text { SPARC (Secreted protein acidic and rich in } \\
\text { cysteine) }\end{array}$ & -0.42 & $-0.71^{* *}$ & $-0.69 *$ & -1.05 & -0.54 \\
\hline ACYPI24889 & Toll-like receptor $7 \dagger$ & 0.01 & $-1.35^{* *}$ & $-1.08 *$ & -1.26 & -0.20 \\
\hline ACYPI000953 & Hydroxysteroid dehydrogenase-like protein 2 & -0.69 & -0.76 ** & $-2.63 *$ & -0.76 & -0.13 \\
\hline ACYPI47960 & MD-2 related lipid-recognition protein-like $†$ & -0.76 & $-1.18^{* *}$ & -0.39 & -0.52 & -0.21 \\
\hline
\end{tabular}

${ }^{* *}$ FDR $<0.05$

${ }^{*}$ FDR $<0.1$

† Uncharacterized in aphid genome v.2; annotation based on blast results. 
760 761 762 763

764

765

766 767 768

\section{P01} difference

Hamiltonella 179 vs Spiroplasma Hamiltonella 445 vs Spiroplasma

Serratia vs Spiroplasma

2.54

313 vs No Symb

$-1.51$

difference

Lower bound

1.44

$-0.01$

$-2.99$

Upper bound

4.53

3.08

0.09

Adjusted p-value

0.0011

0.0513

0.0652

Other Symbiont Species:

No Symb. vs Spiroplasma

Hamiltonella 445 vs Ham. 179

Serratia vs. Hamiltonella .179

No Symb. vs Hamiltonella 179

Serratia vs Hamiltonella 445

No Symb. vs Hamiltonella 445

No Symb. vs Serratia

3.36

3.47

0.37

$-0.18$

0.10

Lower bound

Upper bound

3.47

1.96

$-2.44$

$-0.57$

\section{Adjusted p-value}

$<0.001$

0.031

0.004

\section{Hemocytin}

Hamiltonella 179 vs Spiroplasma

Hamiltonella 445 vs Spiroplasma

Serratia vs Spiroplasma

No Symb. vs Spiroplasma

Hamiltonella 445 vs Ham. 179

Serratia vs. Hamiltonella .179

No Symb. vs Hamiltonella .179

Serratia vs Hamiltonella .445

No Symb. vs Hamiltonella .445

No Symb. vs Serratia
$-3.00$

$-3.54$

$-3.10$

$-3.65$

$-0.54$

difference

$-0.29$

$-0.79$

0.03

$-0.29$

$-0.50$

0.33

0.00

0.83

0.50

$-0.32$

Lower bound

2.57

2.68

$-0.34$

$-0.97$

$-0.76$

$-3.79$

$-4.41$

$-3.89$

$-4.51$

$-1.34$

Lower bound

$-0.99$

$-1.50$

$-0.59$

$-0.99$

$-1.27$

$-0.38$

$-0.77$

0.12

$-0.27$

$-1.02$
Upper bound

4.15

4.26

1.07

0.61

0.97

$-2.21$

$-2.68$

$-2.31$

$-2.78$

0.24

Upper bound

0.41

$-0.09$

0.66

0.42

0.27

1.03

0.78

1.53

1.28

0.39
Adjusted p-value

$<0.001$

$<0.001$

0.54

0.96

0.99

$<0.001$

$<0.001$

$<0.001$

$<0.001$

0.27 
772 Table S3: Post-hoc tests (Tukey's HSD) of Regiella densities in F1 lines

773 774

\begin{tabular}{lllll} 
Comparison & difference & Lower bound & Upper bound & Adjusted p-value \\
\hline 663 vs $(663 \times 317 . A)$ & 0.91 & 0.02 & 1.84 & 0.056 \\
663 vs $(663 \times 317 . B)$ & 1.22 & 0.07 & 2.38 & $\mathbf{0 . 0 3 3}$ \\
663 vs $(663 \times 317 . D)$ & 1.30 & 0.38 & 2.23 & $\mathbf{0 . 0 0 3}{ }^{* *}$ \\
663 vs $(317 \times 663 . A)$ & 1.45 & 0.52 & 2.37 & $<0.001^{* * *}$ \\
663 vs $(317 \times 663 . B)$ & 1.19 & 0.38 & 2.00 & $\mathbf{0 . 0 0 2} * *$ \\
663 vs 317 & 2.38 & 1.37 & 3.38 & $<0.001^{* * *}$ \\
$(663 \times 317 . A)$ vs $(663 \times 317 . B)$ & 0.31 & -0.89 & 1.51 & 0.98 \\
$(663 \times 317 . A)$ vs $(663 \times 317 . D)$ & 0.39 & -0.58 & 1.37 & 0.84 \\
$(663 \times 317 . A)$ vs $(317 \times 663 . A)$ & 0.54 & -0.44 & 1.51 & 0.58 \\
$(663 \times 317 . A)$ vs $(317 \times 663 . B)$ & 0.28 & -0.58 & 1.15 & 0.94 \\
$(663 \times 317 . A)$ vs 317 & 1.46 & 0.41 & 2.52 & $\mathbf{0 . 0 0 3}$ ** \\
$(663 \times 317 . B)$ vs $(663 \times 317 . D)$ & 0.08 & -1.11 & 1.28 & $>0.99$ \\
$(663 \times 317 . B)$ vs $(317 \times 663 . A)$ & 0.23 & -0.97 & 1.42 & $>0.99$ \\
$(663 \times 317 . B)$ vs $(317 \times 663 . B)$ & -0.03 & -1.14 & 1.08 & $>0.99$ \\
$(663 \times 317 . B)$ vs 317 & 1.15 & -0.11 & 2.41 & 0.088 \\
$(663 \times 317 . D)$ vs $(317 \times 663 . A)$ & 0.14 & -0.83 & 1.12 & $>0.99$ \\
$(663 \times 317 . D)$ vs $(317 \times 663 . B)$ & -0.11 & -0.98 & 0.76 & $>0.99$ \\
$(663 \times 317 . D)$ vs 317 & 1.07 & 0.02 & 2.13 & $\mathbf{0 . 0 4 5}$ \\
$(317 \times 663 . A)$ vs $(317 \times 663 . B)$ & -0.25 & -1.11 & 0.61 & $>0.99$ \\
$(317 \times 663 . A)$ vs 317 & 0.93 & -0.13 & 1.98 & 0.11 \\
$(317 \times 663 . B)$ vs 317 & 1.18 & 0.23 & 2.14 & $\mathbf{0 . 0 0 9} * *$
\end{tabular}


776 777 778 779 780

781

782

783

Gene: P01

663 vs 317

663 vs $(663 \times 317)$

663 vs $(317 \times 663)$

317 vs $(663 \times 317)$

317 vs (317x663)

(663x317) vs (317x663)

Difference

2.58

0.99

1.82

$-1.60$

$-0.77$

0.83

Difference

Gene: PO2

663 vs 317

663 vs $(663 \times 317)$

663 vs $(317 \times 663)$

317 vs $(663 \times 317)$

317 vs (317x663)

$(663 \times 317)$ vs $(317 \times 663)$

2.72

1.38

2.00

$-1.34$

$-0.72$

0.61

Gene: Hemocytin

$$
663 \text { vs } 317
$$

663 vs $(663 \times 317)$

663 vs $(317 \times 663)$

317 vs $(663 \times 317)$

317 vs (317x663)

(663x317) vs $(317 \times 663)$

Difference

1.38

1.37

1.82

$-0.01$

0.45

0.46

Difference

Gene: NOS

1.77

0.81

1.35

$-0.96$

$-0.42$

785

663 vs $(663 \times 317)$

663 vs $(317 \times 663)$

317 vs $(663 \times 317)$

317 vs (317x663)

$(663 \times 317)$ vs $(317 \times 663)$

0.53

Lower bound
0.42
0.40
0.40
0.41
0.41
0.40

Upper bound

6.12

2.45

4.52

$-3.88$

$-1.86$

2.10

Lower bound

0.41

0.39

0.39

0.40

0.40

0.39

Lower bound

0.59

0.57

0.59

0.58

0.60

0.58

Lower bound

0.77
0.74

0.74

0.74

0.75

0.75

0.72

Upper bound

6.64

3.50

5.06

$-3.32$

$-1.80$

1.59

Adjusted p-value

$<0.001^{\text {*** }}$

0.095

$<0.001^{* * *}$

0.0039 **

0.26

0.18 
787 Table S5: Collection information for aphid genotypes.

788

789

Host Genotype (lab code)

LSR Ithaca, NY, USA

317

133

663
Glouchestershire, UK

Berkshire, UK

Oxfordshire, UK

\section{Year Collected Symbionts \\ Biotype}

1998
2003
2012
2014

Reg

Reg

$\mathrm{Ham}$

None
Medicago sativa

Trifolium pratensae

Ononis spinosa

Lotus corniculatus

791

792 
793 Table S6: Collection information for symbiont strains.

794

\begin{tabular}{|c|c|c|}
\hline $\begin{array}{l}\text { Symbiont } \\
\text { Genotype } \\
\text { (lab code) }\end{array}$ & Species & Location Collected \\
\hline
\end{tabular}

\begin{tabular}{llll}
\hline .LSR & Regiella insecticola (clade 1) & Ithaca, NY, USA & 1998 \\
.313 & Regiella insecticola (clade 2) & Gloucestershire, UK & 2007 \\
.161 & Spiroplasma sp. & Oxfordshire, UK & 2006 \\
.179 & Hamiltonella defensa & Ithaca, NY, USA & 2015 \\
.445 & Hamiltonella defensa & Ithaca, NY, USA & 2015 \\
.509 & Serratia symbiotica & Knoxville, TN, USA & 2019
\end{tabular}


Screening for secondary symbiont infections:
Regiella

Serratia

Spiroplasma

Hamiltonella

AGTTTGATCATGGCTCAGATTG
AGAGTTTGATCMTGGCTCAG
ATTCTTCAGTAAAAATGCTTGGA
AGTTTGATCATGGCTCAGATTG

Regiella MLST sequencing:

\section{CAYATGSGCATCTCTGCC} AAAACATTGTCTTCCGGG
CGCATTGGGAGAAAAGCCAAG

\author{
GGTAACGTCAATCGATAAGCA \\ TTTGAGTTCCCGACTTTATCG \\ ACACATTTACTTCATGCTATTGA \\ AAATGGTATTSGCATTTATCG
}

\section{Regiella density via qPCR}

Regiella hrpA NAi

Gene knockdown via RNAi

\begin{tabular}{ll} 
lacZ & TAATACGACTCACTATAGGG \\
PO1 & AGACCACACCATGATTACGCCAAGCTC \\
(ACYPI004484) & TAATACGACTCACTATAGGG \\
Hemocytin & CGAGCTACTGCGGTATCCTT \\
(ACYPI003478) & TAATACGACTCACTATAGGG \\
\hline
\end{tabular}

Immune gene expression using qPCR:
AATTCACTACTTTGAAAACCCGG

TTTTCAAARTTNAGCAARTCMGG

TAATACGACTCACTATAGGG

AGACCACCATATCGGTGGTCATCATGC (72) TAATACGACTCACTATAGGG ACATTATTGGTGTTTGCGAATG TAATACGACTCACTATAGGG
CGGGAATTTCATTGAACGAC

CGAGGAGAACATGCTCTTAGAC

GGCCAAGGGTCATTACACTGA

CAAAGTGATCGTTATGACAAACTCAA

CACTGTCCGTAGCATTGAT

ACGTGCGTATACGTTTCTCGAA

ACAATTCGgCGTAAAGGAGGT
TAGTGCTATCGGCAAACGGT
CCTTCCACCAAGCCATGACG This study AGGCCAACCTTGTTCTACTCC
This study

This study
*T7 promoter sequence shown in bold

\begin{tabular}{|c|c|c|c|}
\hline $\begin{array}{l}\text { G3PDH } \\
\text { (ACYPI009769) }\end{array}$ & CGGGAATTTCATTGAACGAC & TCCACAACACGGTTGGAGTA & (73) \\
\hline $\begin{array}{l}\text { NADH } \\
\text { (ACYPI009382) }\end{array}$ & CGAGGAGAACATGCTCTTAGAC & GATAGCTTGGGCTGGACATATAG & (73) \\
\hline $\begin{array}{l}\beta \text {-tubulin } \\
\text { (ACYPI001007) }\end{array}$ & GGCCAAGGGTCATTACACTGA & TGCGAACCACGTCCAACA & $(30)$ \\
\hline $\begin{array}{l}\text { Rpl32 } \\
\text { (ACYPI000074) }\end{array}$ & CAAAGTGATCGTTATGACAAACTCAA & CGTCTTCGGACTCTGTTGTCAA & $(30)$ \\
\hline $\begin{array}{l}\text { PO1 } \\
\text { (ACYPI004484) }\end{array}$ & CACTGTCCGTAGCATTGAT & GGCAGAATAATCGTGAGGTA & $(63)$ \\
\hline $\begin{array}{l}\text { PO2 } \\
\text { (ACYPI072244) }\end{array}$ & ACGTGCGTATACGTTTCTCGAA & TGGCTTCCTATTCTGTTTTGCA & This study \\
\hline $\begin{array}{l}\text { Hemocytin } \\
\text { (ACYPI003478) }\end{array}$ & ACAATTCGGCGTAAAGGAGGT & TGGCATGTAATCGACGGTGT & This study \\
\hline $\begin{array}{l}\text { NOS } \\
\text { (ACYPI001689) }\end{array}$ & TAGTGCTATCGGCAAACGGT & CGGATACTGCGGGAAGACAG & This study \\
\hline
\end{tabular}


Table S8: Sequencing and alignment results

\begin{tabular}{|c|c|c|c|c|}
\hline Host Genotype & Treatment & $\begin{array}{l}\text { Read Pairs (after } \\
\text { QC) }\end{array}$ & Map Rate & $\begin{array}{c}\text { Read pairs } \\
\text { mapped to an } \\
\text { exon }\end{array}$ \\
\hline LSR1 & Control A & $26,834,362$ & $\begin{array}{l}\text { L: } 90.3 \% \\
\text { R: } 89.7 \%\end{array}$ & $22,247,678$ \\
\hline LSR1 & Control B & $23,898,256$ & $\begin{array}{l}\text { L: } 89.0 \% \\
\text { R: } 88.4 \%\end{array}$ & $20,040,867$ \\
\hline LSR1 & Control C & $20,567,298$ & $\begin{array}{l}\text { L: } 90.1 \% \\
\text { R: } 89.5 \%\end{array}$ & $17,513,945$ \\
\hline LSR1 & Control D & $30,839,554$ & $\begin{array}{l}\text { L: } 90.5 \% \\
\text { R: } 89.4 \%\end{array}$ & $26,232,845$ \\
\hline LSR1 & + Clade 1 (.LSR) A & $25,834,362$ & $\begin{array}{l}\text { L: } 90.3 \% \\
\text { R: } 89.7 \%\end{array}$ & $22,247,678$ \\
\hline LSR1 & + Clade 1 (.LSR) B & $22,145,457$ & $\begin{array}{l}\text { L: } 89.1 \% \\
\text { R: } 88.1 \%\end{array}$ & $18,466,505$ \\
\hline LSR1 & + Clade 1 (.LSR) C & $27,986,917$ & $\begin{array}{l}\text { L: } 89.4 \% \\
\text { R: } 89.1 \%\end{array}$ & $23,737,329$ \\
\hline LSR1 & + Clade 1 (.LSR) D & $21,490,058$ & $\begin{array}{l}\text { L: } 89.8 \% \\
\text { R: } 89.3 \%\end{array}$ & $18,248,263$ \\
\hline LSR1 & + Clade $2(.313) \mathrm{A}$ & $20,099,181$ & $\begin{array}{l}\text { L: } 89.2 \% \\
\text { R: } 88.5 \%\end{array}$ & $16,863,392$ \\
\hline LSR1 & + Clade $2(.313) \mathrm{B}$ & $19,020,667$ & $\begin{array}{l}\text { L: } 88.9 \% \\
\text { R: } 88.5 \%\end{array}$ & $15,942,798$ \\
\hline LSR1 & + Clade $2(.313) \mathrm{C}$ & $21,612,263$ & $\begin{array}{l}\text { L: } 89.7 \% \\
\text { R: } 89.0 \%\end{array}$ & $18,295,757$ \\
\hline LSR1 & + Clade $2(.313) \mathrm{D}$ & $23,324,084$ & $\begin{array}{l}\text { L: } 89.6 \% \\
\text { R: } 88.6 \%\end{array}$ & $19,704,560$ \\
\hline Lotus corniculatus & Control A & $28,664,619$ & $\begin{array}{l}\text { L: } 86.7 \% \\
\text { R: } 86.1 \%\end{array}$ & $23,297,559$ \\
\hline Lotus corniculatus & Control B & $32,207,455$ & $\begin{array}{l}\text { L: } 87.5 \% \\
\text { R: } 86.8 \%\end{array}$ & $25,222,986$ \\
\hline Lotus corniculatus & Control C & $25,987,704$ & $\begin{array}{l}\text { L: } 86.7 \% \\
\text { R: } 86.3 \%\end{array}$ & $21,067,089$ \\
\hline Lotus corniculatus & Regiella A & $24,420,268$ & $\begin{array}{l}\text { L: } 87.1 \% \\
\text { R: } 86.7 \%\end{array}$ & $19,780,712$ \\
\hline Lotus corniculatus & Regiella B & $22,622,062$ & $\begin{array}{l}\text { L: } 87.7 \% \\
\text { R: } 87.2 \%\end{array}$ & $18,485,841$ \\
\hline Lotus corniculatus & Regiella C & $30,883,446$ & $\begin{array}{l}\text { L: } 87.3 \% \\
\text { R: } 86.8 \%\end{array}$ & $25,106,295$ \\
\hline Ononis spinosa & Control A & $25,428,345$ & $\begin{array}{l}\text { L: } 87.6 \% \\
\text { R: } 86.9 \%\end{array}$ & $20,652,853$ \\
\hline Ononis spinosa & Control B & $24,322,991$ & $\begin{array}{l}\text { L: } 87.8 \% \\
\text { R: } 87.2 \%\end{array}$ & $19,823,044$ \\
\hline Ononis spinosa & Control C & $30,277,729$ & $\begin{array}{l}\text { L: } 87.7 \% \\
\text { R: } 87.2 \%\end{array}$ & $24,579,747$ \\
\hline Ononis spinosa & Regiella A & $26,677,905$ & $\begin{array}{l}\text { L: } 87.9 \% \\
\text { R: } 87.3 \%\end{array}$ & $21,741,656$ \\
\hline Ononis spinosa & Regiella B & $29,108,115$ & $\begin{array}{l}\text { L: } 87.8 \% \\
\text { R: } 87.3 \%\end{array}$ & $23,498,863$ \\
\hline Ononis spinosa & Regiella C & $25,669,486$ & $\begin{array}{l}\text { L: } 87.8 \% \\
\text { R: } 86.4 \%\end{array}$ & $20,955,594$ \\
\hline Trifolium pratense & Control A & $22,440,188$ & $\begin{array}{l}\mathrm{L}: 86.7 \% \\
\mathrm{R}: 85.9 \%\end{array}$ & $17,919,579$ \\
\hline Trifolium pretense & Control B & $20,932,628$ & $\begin{array}{l}\text { L: } 86.8 \% \\
\text { R: } 85.9 \%\end{array}$ & $16,741,330$ \\
\hline Trifolium pretense & Control C & $22,905,550$ & $\begin{array}{l}\text { L: } 86.6 \% \\
\text { R: } 85.6 \%\end{array}$ & $18,228,176$ \\
\hline Trifolium pretense & Regiella A & $27,502,459$ & $\begin{array}{l}\text { L: } 87.8 \% \\
\text { R: } 87.3 \%\end{array}$ & $22,631,920$ \\
\hline Trifolium pretense & Regiella B & $20,253,813$ & $\begin{array}{l}\text { L: } 87.0 \% \\
\text { R: } 86.7 \%\end{array}$ & $16,395,529$ \\
\hline Trifolium pratense & Regiella C & $23,107,988$ & $\begin{array}{l}\text { L: } 86.3 \% \\
\text { R: } 85.2 \%\end{array}$ & $18,251,833$ \\
\hline
\end{tabular}

